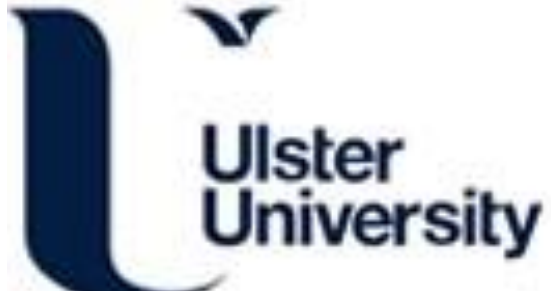

\section{Waves and wrecks: A computational fluid dynamic study in an underwater archaeological site}

Fernández-Montblanc, T., Izquierdo, A., Quinn, R., \& Bethencourt, M. (2018). Waves and wrecks: A computational fluid dynamic study in an underwater archaeological site. Ocean Engineering, 163, $232-250$. https://doi.org/10.1016/j.oceaneng.2018.05.062

Link to publication record in Ulster University Research Portal

\section{Published in: \\ Ocean Engineering}

Publication Status:

Published (in print/issue): 01/09/2018

DOI:

10.1016/j.oceaneng.2018.05.062

\section{Document Version}

Author Accepted version

\section{General rights}

Copyright for the publications made accessible via Ulster University's Research Portal is retained by the author(s) and / or other copyright owners and it is a condition of accessing these publications that users recognise and abide by the legal requirements associated with these rights.

\section{Take down policy}

The Research Portal is Ulster University's institutional repository that provides access to Ulster's research outputs. Every effort has been made to ensure that content in the Research Portal does not infringe any person's rights, or applicable UK laws. If you discover content in the Research Portal that you believe breaches copyright or violates any law, please contact pure-support@ulster.ac.uk. 


\section{Waves and wrecks: A computational fluid dynamic \\ 2 study in an underwater archaeological site}

3 Fernández-Montblanc, T. ${ }^{a}$, Izquierdo, A. ${ }^{\text {, }}$ Quinn, R. ${ }^{\text {, }}$ Bethencourt, M. ${ }^{d}$

4 a Department of Applied Physics, International Campus of Excellence of the

5 Sea (CEI-MAR), University of Cádiz, Avda. República Saharaui s/n, 11510,

6 Puerto Real, Cádiz, Spain. tomas.fernandez@uca.es. (Corresponding author)

$7{ }^{b}$ Department of Applied Physics, International Campus of Excellence of the

8 Sea (CEI.MAR), University of Cádiz, Avda. República Saharaui s/n, 11510,

9 Puerto Real, Cádiz, Spain. alfredo.izquierdo@uca.es.

10 ' School of Environmental Sciences, Ulster University, Coleraine, BT52 1SA,

11 Northen Ireland. rj.quinn@ulster.ac.uk.

$12{ }^{d}$ Department of Materials Science, Metallurgy Engineering and Inorganic

13 Chemistry, International Campus of Excellence of the Sea (CEI.MAR),

14 University of Cádiz, Avda. República Saharaui s/n, 11510, Puerto Real, Cádiz,

15 Spain. manuel.bethencourt@uca.es.

17 Abstract

18 The modification of waves by shipwrecks and the resulting scour play

19 important roles in shipwreck site formation, and conservation of

20 archaeological sites. The oscillatory flow induced by waves and its

21 interaction with the hull structure at a historic shipwreck site was analyzed

22 using a two phase 2D model based on the Reynolds averaged Navier-Stokes

23 equations and shear stress transport (SST) k-Omega turbulence model,

24 with inputs from field-based bathymetric survey. The relative importance 
and seasonal variation in hydrodynamic processes were investigated (flow

velocity increase, coherent structures and vortex shedding, turbulence and

27 steady current induced by the non linearity of waves). Results demonstrate

28 that frictional velocity and flow increase dominate morphological change in the low wave energy period (LEP), whereas turbulent shear stress and large coherent structures dominate scouring in the high wave energy period

31 (HEP). Furthermore, flow acceleration around the hull structure and recirculation cells originated by wave non-linearities become more prominent in the HEP, modifying the trajectory of the shedding vortex and increasing its capacity to transport sediment. The results demonstrate, for the first time, that computational fluid dynamics is a valuable tool in assessing the wave structure interaction in full scale and realistic morphological conditions at complex shipwreck sites.

Keywords: Computational fluid dynamics; Multi-beam echo-sounder; Shipwreck; Site formation processes; Hydrodynamics; scouring

\section{Introduction}

41 In the last decades, advances in technology have led to the development,

42 exploitation and exploration of the seabed resulting in the discovery of a

43 considerable number of shipwrecks and other archaeological sites

44 (Besrgstrand and Godfrey, 2007). Simultaneously, marine environmental conditions and their relationship to underwater cultural heritage $(\mathrm{UCH})$ have provoked considerable interest due to the direct link to the status of

47 underwater cultural heritage and the perspective of conservation in situ 48 (Leino, 2011; Wheeler, 2002). In particular, physical processes have been 49 proposed by various authors as dominant in early stages of site formation 
50 (Quinn, 2006; Ward et al., 1999), and the coupling between water and

51 sediment dynamics controlling the sediment budget is considered as

52 fundamental in site formation studies (Smyth and Quinn, 2014).

53 Quantifying sediment budgets at wreck sites is important, as sediment

54 offers physical stability to shipwrecks, and the deposition and erosion of

55 sediment at sites modifies the geochemical characteristics which control

56 chemical degradation and the biological activity influencing the decay of

57 UCH materials (Ruuskanen et al., 2015;Ward, 1999).

58 Sediment budgets at shipwreck sites depend on the sediment availability

59 and characteristics, the hydrodynamic regime, and its interaction with the

60 shipwreck structure. Scour takes place when an obstacle modifies the flow

61 pattern in its immediate neighbourhood causing an increase in local

62 sediment transport. Given the importance of scour in the sediment budget

63 around shipwrecks and in site formation and conservation studies, the

64 scouring process has received increased attention from the scientific

65 community in the last decade (e.g. Astley et al., 2016; Baeye et al., 2016;

66 Fernández-Montblanc et al., 2016; Smyth and Quinn, 2014; Quinn, 2006;

67 Quinn and Smyth, in press).

68 Thus, several research projects have focused on understanding shipwrecks

69 under steady flow conditions, including sediment dynamics and associated

70 scour pit formation. These studies used different data and methodologies,

71 and can be subdivided by type into accretion-erosion models (Astley et al.,

72 2016; Bates et al., 2011; Quinn and Boland, 2010), scaled physical models

73 (Saunders, 2005) and computational fluid dynamics (CFD) models in

74 combination with high resolution multibeam echosounder bathymetric data

75 (Smyth and Quinn, 2014; Quinn and Smyth, in press). 
However, the fate of shipwrecks dominated by oscillatory flow and

77 associated scouring has been considerably less studied. A real-world case

78 study of shipwreck scouring under shoaling waves based on side scan sonar

79 data is outlined in Quinn (2006). The burial-exposure of different artefacts

80 in an archaeological context is described in McNinch et al. (2006) using

81 numerical modelling, calibrated by sediment and hydrodynamic

82 observations. More recently, wave propagation and hydrodynamic modelling

83 in combination with time-lapse bathymetric surveys and hydrodynamic

measurements was used to characterize scour and seasonal

morphodynamic changes in an energetic shallow water shipwreck site where part of the hull has been preserved (Fernandez-Montblanc et al., 2016).

87 Conversely, the scouring due to wave and obstacle interaction has been widely studied in other scientific fields such as coastal engineering, with applications to breakwaters (Sumer et al.; 2001, 2005, Young and Testik, 2009), pipelines and mines (Mattioli et al., 2013; Sumer, 1990; Voropayev,

91 et al., 2003), or offshore structures mounted on the seabed (Nielsen,

92 2012). As a result, scouring can be estimated through simple empirical

93 equations. However, those studies have been performed usually assessing

94 the flow interaction with relative simple geometries, as those are commonly used in coastal and offshore engineering. The majority of these studies are based on scaled physical models with inherent limitations. Therefore, due to

97 its importance in site formation and in-situ conservation of $\mathrm{UCH}$, there is a need to further investigate the oscillatory flow interaction with complex geometrical structures (i.e. shipwrecks). It is important for these models to incorporate real and complex morphological scenarios to reflect those found

101 in nature, and to use field data to calibrate and validate them. 
102 In this context, CFD represents a reliable and cost-effective way of

103 calculating the flow pattern around complex geometrical structures.

104 Increasing computing power and resulting simulation efficiency allows the

105 application of CFD to large domains representing complex three dimensional

106 seabed structures, natural or man-made, where the scour and

107 morphodynamic behaviour are associated with near-bed turbulent flow. This

108 study focuses on the application of CFD to investigate the interaction

109 between a historic shipwreck and wave-induced oscillatory flow, with the

110 aim of assessing the relative importance of different mechanisms that

111 originate and control scouring in these environments. CFD modelling is

112 validated with field-based hydrodynamic data.

\section{Theoretical background}

115 An object placed on the seabed changes the flow pattern in the immediate

116 vicinity. Changes may result in flow contraction, promoting streamline

117 convergence and flow acceleration, vortex formation around the structure

118 (with or without vortex shedding behind the structure), increased

119 turbulence, and modification of wave characteristics (occurrence of

120 reflection and diffraction or breaking). As a result, shear stress is increased

121 on the seabed, promoting sediment transport around the object and leading

122 to scour (Sumer and Fredsøe, 2002). The process continues until a new

123 quasi-equilibrium state is reached, in which the increase in bed shear stress

124 due to the presence of the structure is balanced as a direct consequence of

125 depth increment and/or the loss of fine sediment in the scoured area. If the

126 eroding seabed is composed of fined-grained (clay-silt) material, suspension 
127 transport carries the sediment far away from the structure (Whitehouse et

128 al., 2011b), but if the seabed is composed of coarse-grained (sand-gravel)

129 sediment, the sediment is deposited near the structure (Smyth and Quinn, 130 2014).

131 Oscillatory flow adds time-dependence to the above phenomena. The wave

132 boundary layer, horizontal and vertical velocity changes, turbulence, and

133 the large coherent structures (LCS) formed around the object vary along a 134 wave cycle. Studies on oscillatory flow around submerged breakwaters and 135 horizontal cylinders on the seabed indicate that the flow pattern and scour 136 are mainly controlled by the Keulegan-Carpenter (KC) number (Vorapayev, 137 2003):

$138 K C=2 \pi a / D$,

139 where $D$ is the characteristic length of the object (i.e. diameter for a 140 cylinder), $a=U_{m o} T / 2 \pi$ is the amplitude of the free stream motion, and $\mathrm{T}$ is 141 the wave period. Sumer (1991) analysed the vortex dynamics around a 142 horizontal cylinder on the wall at high Reynolds (Re) numbers (mostly at $\left.143 \operatorname{Re} \sim 10^{5}\right):$

$144 \operatorname{Re}=U_{m o} D / v$,

145 where $v$ is the kinematic viscosity of water and $U_{m o}$ the maximum value of 146 the free stream velocity. Sumer (1991) found that for small values of $K C$ no 147 flow separation occurs. However, increasing $K C$ to 4 promotes flow 148 separation and symmetrical vortex formation upwave and downwave of the 149 cylinder, and for $4>K C>7$, asymmetry in the formation and motion of the 150 vortices is introduced into the system. At $K C>7$, vortex shedding occurs. 151 Similar results were found in a physical experiment with sinusoidal forcing 
152 at $\operatorname{Re}=300-500$, carried out by Testik et al. (2005). They found horseshoe

153 vortices of opposite rotation formed periodically on both sides of the

154 cylinder. For $K C=8.4$, the vortex formed on each side washed over the

155 cylinder in the following half cycle and formed a vortex pair with the initial

156 one (with the opposite vorticity sign) and shed away periodically as a paired

157 structure. In the case of physical experiments forced with waves, instead of

158 sinusoidal forcing, no shedding of the paired structure was found in

159 experiments with $7>K C>23$ and Re ranging from $310^{3}$ to $2.610^{4}$ (Mattioli

160 et al., 2013). Investigations on scour around vertical piles exposed to waves

161 have shown the increment in bed shear stress due to horseshoe vortex

162 development, especially in the turbulent bottom boundary layer (Sumer et

163 al., 1997). Although the onset of scour is related to the lee-wake vortex and

164 vortex shedding, flow sweeps the sediment into the core and the shedding

165 vortex carries the sediment away from the pile as it is advected

166 downstream (Sumer et al., 1993). A more recent scour study around a half-

167 buried sphere under steady flow showed that the increase in turbulence

168 beneath the horseshoe vortex enhances bed shear stress amplification and

169 has a notable contribution to the scour (Dixen, 2013).

170 In addition, the steady current generated by nonlinear effects induced by

171 waves results in a secondary flow in the form of steady streaming and

172 undertow. The steady streaming flow generated by wave propagation over

173 the structure is the result of the non-uniform oscillatory action in presence

174 of the structure. Its relevance in the scouring process has been revealed for

175 a vertical pile and oscillatory flow (Sumer and Fredsoe, 2001) and in a

176 submerged breakwater (Sumer et al., 2005). Steady streaming $O\left(10^{-1} \mathrm{~m} \cdot \mathrm{s}^{-}\right.$

$\left.177^{1}\right)$ transports and redistributes the sediment that the oscillatory flow $O\left(10^{0}\right.$ 
$178 \mathrm{~m} \cdot \mathrm{s}-1$ ) brings into suspension. Also the non uniformity in vertical

179 distribution of spanwise velocity generates a steady flow known as

180 undertow; a gravity driven current with the maximum located near the

181 seabed and oriented seaward. Undertow is induced by two mechanisms: (1)

182 rollers that carry water shoreward in the upper water column under

183 breaking conditions, and (2) eulerian drift in the case of non-breaking

184 conditions.

185 Finally, various authors have noted the increment in sediment transport

186 produced by turbulence, whether internally generated turbulence (Nelson,

187 1995), or externally generated due to flow structure-interaction (Sumer,

188 2003). The turbulence makes the transport of momentum more effective

189 (as well as mass and energy) between fluid layers (Dixen, 2013) with a

190 consequent increment in sediment transport. In addition, turbulence

191 promotes the settling delay (Murray, 1970), which favours the sediment

192 transport in suspension and increases scour.

193 All near wall processes and characteristics, such as the boundary layer

194 structure, thickness, the point of vortex and flow separation, friction

195 velocity and turbulence are conditioned by the roughness of the seabed

196 (sand-grain and bed form roughness) and the roughness characteristics of

197 the of the obstacle.

\section{Methodology}

\section{$199 \quad 3.1$ Study site}

200 Fougueux, a 74-gun Temeraire-class French ship-of-the-line, took part in

201 the Battle of Trafalgar in October 1805, where the British fleet defeated the

202 combined French-Spanish fleet off the coast of Cádiz. Fougueux was 
203 wrecked after the battle, when she was driven onto a rocky shoal. The

204 wreck site is characterized by low to moderate wave energy conditions, with

205 the exception of high the energetic winter storms from WSW. Partial burial

206 in sediment has resulted in the preservation of a considerable portion of the

207 wooden hull structure $\left(15 \times 7 \mathrm{~m}^{2}\right)$ along with 31 cannons and a large anchor

208 (Rodríguez Mariscal et al., 2010).

209 The Fougueux wreck site is located on the south-western seashore of the

210 Iberian Peninsula (Gulf of Cádiz), in a shoal seaward of the Sancti Petri sand

211 spit (Figure 1a). The seafloor around the wreck is gently sloping from 5-10

212 m depth (Figure 1a), and comprises unconsolidated well-sorted fine quartz

213 sand $\left(D_{50}=1.8 \cdot 10^{-4} \mathrm{~m}\right)$ with bedrock areas where Plio-quaternary shelly

214 conglomerate crops out (Gracia et al., 2008). The scattered wreck remains

215 are located in an energetic shallow-water environment, where wave

216 oscillatory flow promotes scouring resulting in inshore pits (maximum depth

217 of $0.77 \mathrm{~m}$ ) scoured deeper than offshore pits (0.35 m depth) (Figure $1 \mathrm{a})$.

218 Seasonal changes are observed, showing accretion during a low wave-

219 energy period (LEP) from May to September and net erosion around the hull

220 structure during a high-energy period (HEP) from November to April

221 (Fernández-Montblanc et al., 2016). At the deepest point at the centre of

222 the wreck site $(x \sim=192)$ minor seasonal variations were found (Figure 1a

223 and Figure 1c). The mean wave height at the site is $0.67 \mathrm{~m}$ during LEP and

$2241.18 \mathrm{~m}$ in HEP, whereas the maximum wave height is $4 \mathrm{~m}$, and is limited by

225 local depth. Mean bottom orbital velocity fluctuates from $0.30 \mathrm{~m} \cdot \mathrm{s}^{-1}$ during

226 LEP to $0.68 \mathrm{~m} \cdot \mathrm{s}^{-1}$ during HEP. The maximum value of near-bed wave orbital

227 velocity, calculated by a wave propagation model, rises to $2 \mathrm{~m} \cdot \mathrm{s}^{-1}$ during

228 winter storms. The area is meso-tidal (mean tidal spring range of $2.96 \mathrm{~m}$ ), 
229 with a dominant semidiurnal tidal variability. Peak currents are orientated

230 NNW and SSE at flood and ebb tide conditions respectively, with mean

231 values $0.15-0.25 \mathrm{~m} \cdot \mathrm{s}^{-1}$. Velocities above this value, directed NNW,

232 correspond to a SE wind. In contrast, current velocities greater than 0.25

$233 \mathrm{~m} \cdot \mathrm{s}^{-1}$, directed SSE, are related to wind drift (Fernández-Montblanc et al., 234 2016).

\section{$235 \quad 3.2$ Field data}

\section{Bathymetric data}

237 In order to collect bathymetric data corresponding to the low energy wave

238 period (LEP; summer conditions) and high energy wave period (HEP; winter

239 conditions), two successive surveys were conducted. The LEP survey was

240 performed on 11.09.2013 using an Ohmex Sonarmite v3.0 singlebeam

241 echosounder operating at $235 \mathrm{kHz}$ (sample rate $1 \mathrm{~Hz}$ and theoretical vertical

242 resolution $0.05 \mathrm{~m}$ ). A HEP survey was conducted on 23.11 .2013 , using a

243 Reson Seabat 8124 multibeam echosounder operating at $200 \mathrm{kHz}\left(1.5^{\circ}\right.$

244 beam width, $0.01 \mathrm{~m}$ vertical resolution and $39.89 \mathrm{~Hz}$ sample rate). In both

245 cases, positional data were acquired using an RTK-GPS Leica 1200, from

246 which tidal and wave corrections were taken. Two digital elevation models

247 were derived from the LEP and HEP surveys, and two $250 \mathrm{~m}$ long

248 bathymetric profiles $\left(\mathrm{AA}^{\prime}\right)$ were extracted and used as a bottom boundary in

249 the CFD study (Figure 1b, Figure 1c). 
(a) 尊

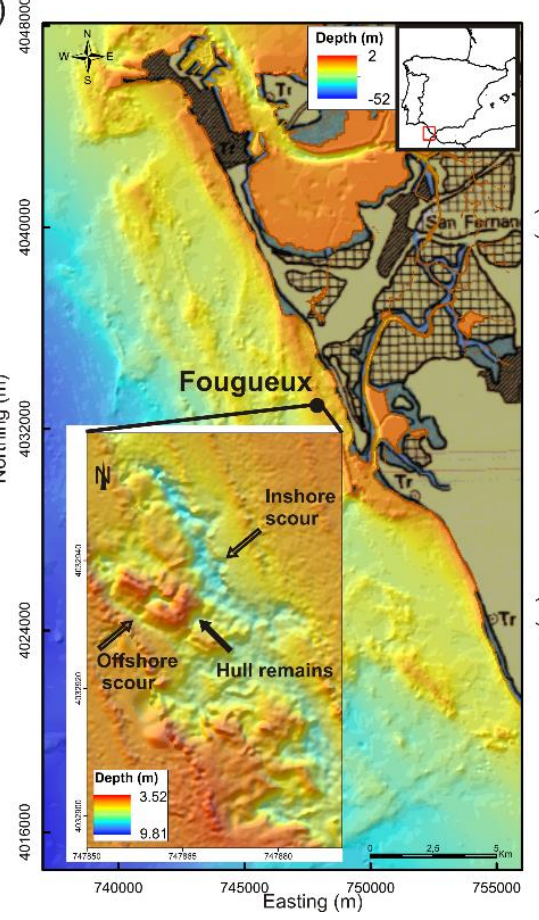

(b)

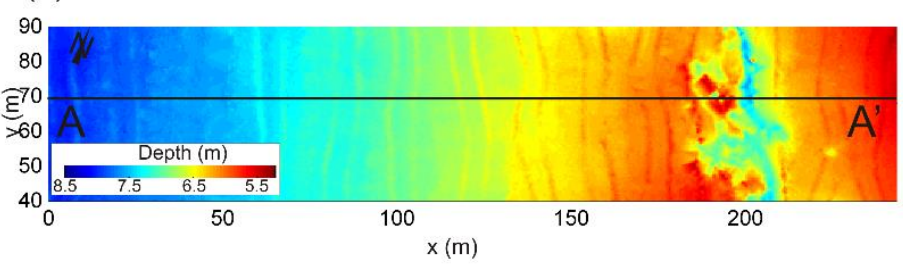

(c)

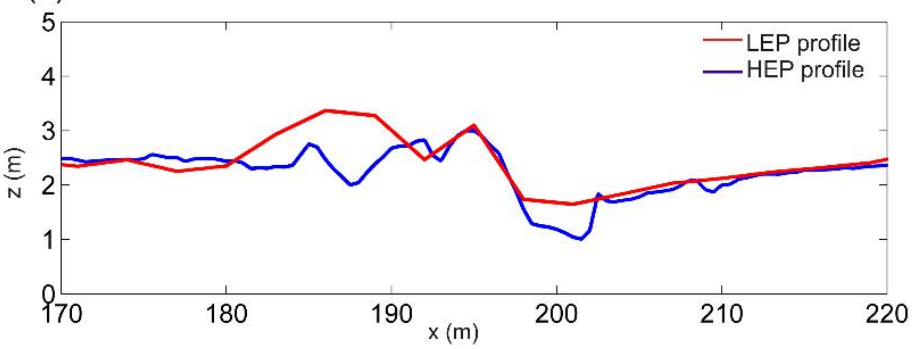

251 Figure 1. (a) Location map of the study site in the Gulf of Cadiz and detail of the

252 Fougueux site from the multibeam bathymetric data. (b) Location of bathymetric

253 profile in multibeam bathymetry. (c) Detail of bathymetric profiles corresponding to

254 LEP and HEP at the Fougueux site.

\section{$255 \quad 3.3$ CFD study}

256 The CFD modelling of the Fougueux site was performed using OpenFOAM $\mathbb{R}$ 257 software, an open source toolbox that allows users to solve problems of 258 turbulence and complex fluid flows using finite volume discretization. The 259 InterFoam solver was employed in this study, solving the three-dimensional 260 Reynolds averaged Navier-Stokes equations for two incompressible phases 261 (sea-water and air in our case represents a free surface flow solver) using a 262 finite volume discretization and the volume of fluid method. Flow turbulence 263 is represented by a two-equation SST K-Omega model (Menter, 1994). This 264 model combines the use of a k-epsilon turbulence model in the free flow 265 regions and a k-omega turbulence model inside the boundary layer, avoiding 266 the problem of the high sensitivity to the inlet boundary condition of the k267 omega model (Bozorgnia et al., 2014). IHFoam was used to implement the 268 boundary conditions for wave generation and absorption, reducing the 
269 problem of reflexion in the boundaries without an extension of the domain 270 (Higuera et al., 2013).

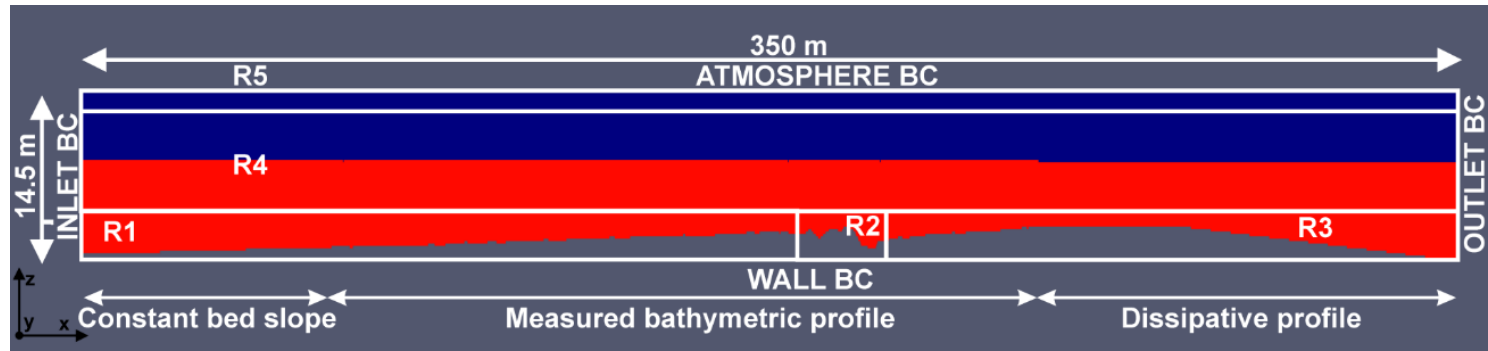

272 Figure 2.Computational domain used in numerical simulation along with boundary

273 conditions (BC).

274 The numerical experiments were carried out in a full scale 2D computational 275 domain, measuring $350.0 \times 14.5 \mathrm{~m}^{2}$ and the reference system was set as 276 illustrated Figure 2. The reference system was placed at the furthest point 277 offshore $(A)$ (Figure $1 \mathrm{~b}$ ). The $\mathrm{x}$ axis is positive in the wave propagation 278 direction ( $\left.A A^{\prime}\right)$ Figure (1b). The $z$ axis for the domain was located at the 279 deepest point furthest offshore, being positive upward (Figure 1c). The 280 model domain was subdivided into regions with different resolutions to 281 reduce the number of cells and computational time (Figure 2). Region R4, 282 where wave propagation takes place, has cell sizes of $\Delta x=0.10 \mathrm{~m}$ and $283 \Delta \mathrm{z}=0.05 \mathrm{~m}$. Region $\mathrm{R} 1$ and $\mathrm{R} 3$ have cell sizes of $\Delta \mathrm{x}=0.10 \mathrm{~m}$ and $\Delta \mathrm{z}$ varying 284 from $0.02 \mathrm{~m}$ at bottom to $0.10 \mathrm{~m}$ at top. Region $\mathrm{R} 2$, where the wreck-wave 285 interaction is focused, comprises an unstructured prismatic mesh of 0.005 $286 \mathrm{~m}$ cell size, allowing the mesh to adapt to the complex geometry of the 287 wreck. The smooth changes in mesh resolution in the transition areas (R1288 R2 and R2-R3) reduces the drastic changes in the near surface boundary 289 flow. The friction coefficient at the seafloor was set as uniform in the whole 290 domain as a rough wall function dependent on Nikuradse roughness length 291 (ks). A grain size value of $D_{50}=1.8 \cdot 10^{-4} \mathrm{~m}$ from measurement on five 
292 sediment samples collected at the shipwreck site was used to calculate a ks

$293=4.5 \cdot 10^{-4} \mathrm{~m}$ using the relation proposed by Soulsby (1997) for a flat bed

294 (ks 2.5 $\cdot \mathrm{D}_{50}$ ). Water density was set equal to $1025 \mathrm{kgm}^{-3}$ corresponding to

$29520^{\circ} \mathrm{C}$ and $35 \mathrm{PSU}$ salinity. Air density was taken as $1 \mathrm{kgm}^{-3}$. The surface

296 tension in the air-water system was given a value of $0.07 \mathrm{kgs}^{-2}$. Numerical

297 simulations were run in parallel on a high performance computing facility

298 using 32 processors $(2.6 \mathrm{GHz})$, of 2 CPUs subdividing the domain into 32

299 subdomains. During the simulation, a variable time step was specified with

300 a Courant number lower than 0.5 ensuring numerical stability.

301 Table 1 outlines the numerical experiments conducted in the study. Five

302 different wave conditions typical of the Fougueux site were simulated using 303 the two bathymetric settings (LEP and HEP). In the first pair of simulations, 304 a case representing mean storm conditions in the area (E1_HEP and 305 E1_LEP) was run. The second pair represents the response to a fading 306 storm (E2_HEP and E2_LEP). Swell wave conditions were simulated for 307 experiment 3 (E3_HEP, E3_LEP), and wave breaking conditions for 308 experiment 4 (E4_HEP, E4_LEP). Finally, a simulation of offshore wave 309 breaking was conducted (E5_HEP, E5_LEP).

310 Assuming that wave propagation takes place along the $\mathrm{x}$ axis which is 311 perpendicular to the shoreline (Figure 1a and Figure 1b), the set of wave 312 parameters (wave phase, wave height and wave periods) were used as 313 inputs at the inflow boundary location (Inlet BC) (Figure 2). IHFoam was 314 used to realistically generate waves at Inlet BC according to different wave 315 theories (see Higuera et al. (2013) for further details). In this set of numerical experiments E1_LEP and E1_HEP were conducted using Stokes II 
317 theory, and cnoidal theory was used for the rest of the experiments,

318 according to the range proposed by Le Méhauté (1976).

\begin{tabular}{|c|c|c|c|c|c|c|c|}
\hline \multirow[b]{2}{*}{ Name } & \multicolumn{4}{|c|}{ Wave characteristics } & \multicolumn{2}{|c|}{ Bathymetry } & \multirow{2}{*}{$\begin{array}{c}\text { Water level } \\
\text { Depth }(\mathrm{m}) \text { on hull } \\
\text { Remains }(x=195 \mathrm{~m}\end{array}$} \\
\hline & $\begin{array}{l}\text { Wave } \\
\text { type }\end{array}$ & $\begin{array}{c}\mathrm{H} \\
(\mathrm{m})\end{array}$ & $\begin{array}{c}\mathrm{T} \\
(\mathrm{s})\end{array}$ & Wave Conditions & HEP & LEP & \\
\hline E1_LEP & Regular & 1.5 & 6 & Mean Storm conditions & & $X$ & 5.52 \\
\hline E1_HEP & Regular & 1.5 & 6 & Mean storm conditions & $X$ & & 5.52 \\
\hline E2_ LEP & Regular & 3.0 & 9 & Fading storm & & $x$ & 6.84 \\
\hline E2_HEP & Regular & 3.0 & 9 & Fading storm & $X$ & & 6.84 \\
\hline E3_ LEP & Regular & 2.4 & 15 & Swell wave conditions & & $x$ & 5.52 \\
\hline E3_HEP & Regular & 2.4 & 15 & Swell wave conditions & $X$ & & 5.52 \\
\hline E4_LEP & Regular & 3.5 & 15 & Wave breaking & & $x$ & 5.52 \\
\hline E4_HEP & Regular & 3.5 & 15 & Wave breaking & $X$ & & 5.52 \\
\hline E5_LEP & Regular & 4.0 & 9 & Offshore wave breaking & & $x$ & 5.52 \\
\hline E5_HEP & Regular & 4.0 & 9 & Offshore wave breaking & $X$ & & 5.52 \\
\hline
\end{tabular}

Table 1. Wave characteristics, bathymetric conditions and water depth used

320 in the numerical experiments.

\section{$321 \quad 3.4 \quad$ Data analysis}

322 The modelled time series of the horizontal and vertical velocity components

323 in all grid nodes were processed to extract the mean and fluctuating terms

324 from instantaneous data, according to

$325 \boldsymbol{u}(x, z, t)=\overline{\boldsymbol{u}}(x, z, t)+\boldsymbol{u}^{\prime}(x, z, t) ;(1)$

326 where $\boldsymbol{u}$ is the instantaneous velocity, $\overline{\boldsymbol{u}}$ is the mean (steady) component

327 and $\boldsymbol{u}^{\prime}$ is the fluctuating (turbulent) component of the instantaneous

328 velocity. Several methods can be applied to separate the mean and

329 turbulent components from an instantaneous velocity (Longo, 2002). These

330 methods include high-pass filtering (Sawamoto et al., 1982), phase

331 averaging (Ting and Kirby,1996), moving phase averaging (Nadaoka,1989;

332 Standy and Feng, 2005), ensemble averaging (Chang and Liu,1999) and

333 differencing method (Scott et al., 2005). In the commonly used phase and 
334 moving phase methods, the wave breaking variation from wave to wave can

335 affect the accuracy of averaging results (Chang and Liu, 1999). Therefore,

336 and considering the high repeatability of the flow field, the ensemble

337 averaging method was applied. To that end the time series of instantaneous

338 velocity $(\boldsymbol{u})$ was divided into wave cycles by applying the zero up crossing

339 method from the free surface elevation signal, where each wave

340 corresponds to the record between two successive zero up crossings, that is

341 when the wave-perturbed sea surface moves upward crossing the zero

342 reference level. Then the ensemble average was computed fixing the time

$343(\hat{t})$ and arithmetically averaging all the $\mathrm{N}$ wave cycles simulated (Eq. 2 ).

344 Subsequently, the random residual turbulent component of the flow was

345 calculated by subtracting the ensemble phase velocity to the instantaneous

346 velocity (Eq.3). Thus, for regular waves the kinematics could be split into

347 periodic component (mean oscillatory flow) with repetitive vortical

348 structures (LCS), and those related to the turbulent motion.

$349\left\langle\boldsymbol{u}(x, z, \hat{t})_{N}\right\rangle=\frac{1}{N} \sum_{n=1}^{N} \boldsymbol{u}_{n}(x, z, \hat{t}) ;(2)$

$350 \quad \boldsymbol{u}^{\prime}(x, z, \hat{t})=\boldsymbol{u}(x, z, \hat{t})-\left\langle\boldsymbol{u}(x, z, \hat{t})_{N}\right\rangle$

351 In order to evaluate the number of wave cycles required to construct the

352 averaged ensemble, convergence tests were performed following the

353 methodological approach in Melville et al. (2002) for the square mean

354 velocity and the turbulent kinetic energy. The tests included all

355 morphological scenarios and wave conditions, and demonstrated that 10

356 wave cycles are enough to guarantee proper separation of steady and

357 turbulent components. 
360 The mean flow was characterized through the streamwise variation of the

361 horizontal velocity profiles and the bottom boundary layer thickness $(\delta)$,

362 defined as the distance from the bottom to the height of maximum

363 horizontal velocity $\left\langle U_{m o}\right\rangle$ at the wave phase $90^{\circ}$ (Jensen et al., 1989). As

364 well as the oscillatory Reynolds number defined as $R e=a U_{m o} / v$, where

$365\left\langle U_{m o}\right\rangle$ the maximum value of the free stream velocity, $v$ the kinematic

366 viscosity (Jensen et al., 1989), $a=U_{m o} T / 2 \pi$ is the amplitude of the free

367 stream motion where $\mathrm{T}$ is the wave period.

\section{Large coherent structures}

369 LCS are defined as connected, large-scale turbulent fluid parcels with

370 phase-correlated vorticity over their spatial extent. These are a

371 characteristic feature of turbulent shear flows, and are responsible for the

372 large-scale transport of mass, heat and momentum (Hussain, 1983).

373 Although several mechanisms can induce LCS, we are interested in the

374 horseshoe vortex, the lee-wake vortex, and vortex shedding, which are

375 strongly related to the presence of an obstacle in a flow. Vortex dynamics

376 contribute to the scouring process since bed shear increases under the

377 vortexing.

378 The LCS can be separated from the background flow field through the use of

379 an approximated criterion based on the Okubo-Weiss parameter W:

$380 \quad W=S_{n}^{2}+S_{s}^{2}-\omega^{2}(6), S_{n}=d\langle u\rangle / d x-d\langle w\rangle / d z ;(7)$

$381 \quad S_{s}=d\langle w\rangle / d x+d\langle w u\rangle / d z(8), \omega=d\langle w\rangle / d x-d\langle u\rangle / d z$ 
382 where $S_{n}$ is the normal strain, $S_{s}$ is the shear strain, $\omega$ is the vorticity, $\langle u\rangle$ is

383 the ensemble averaged horizontal velocity, and $\langle w\rangle$ is the ensemble

384 averaged vertical velocity.

385 The velocity field can be partitioned into three regions according to the 386 magnitude of this criterion. Rotation dominates regions with $W<-W_{0}$, 387 where the vortices are located. Intermediate regions are characterized by 388 small positive and negative values of $W\left(|W| \leq W_{0}\right)$ where the vorticity and 389 strain rate are balanced. The strain dominated regions, with $W>W_{0}$, are 390 located outside the vortices. The threshold value is $W_{0}=0.2 \sigma_{w}$, where $\sigma_{w}$ is 391 the standard deviation of $W$ in the analyzed domain (Pasquero et al., 2001). 392 The application of this criterion depicts the general structure of the vortex, 393 with an eddy core in a vorticity-dominated inner region, and a circulation 394 cell in the strain-dominated outer region (Elhmaïdi et al., 1993). The core 395 edge is identified by the closed lines with $W=0$. The $W$ parameter sign for 396 the extraction of eddy cores has been successfully employed in complex 397 fluid flows (Jeong and Hussain,1995), oceanography (Isern-Fontanet, 2004) 398 and oscillatory flow analysis (Mattioli et al., 2013).

399 The values of the dimensionless vorticity $\left(\omega^{*}\right.$; computed as $\left.\omega^{*}=\omega T\right)$ and 400 dimensionless Okubo-Weiss parameter $\left(W^{*}\right.$; computed as $\left.W^{*}=W T^{2}\right)$ are 401 calculated from the ensemble averaged velocities.

\section{Turbulence}

403 The streamwise variation in the vertical distribution of Reynolds stresses

$404\left(-\rho \overline{u^{\prime} w^{\prime}}\right)$ was computed under different wave conditions (shoaling 405 progressive waves and breaking waves). The results are analysed with 406 streamwise distribution of bed shear stress and mean flow characteristics. 
407 Steady current generated by nonlinear effects induced by waves

408 (SCw): Undertow and steady streaming

409 The steady current, generated by nonlinear effects induced by waves,

410 includes steady streaming and undertow. This wave-induced steady current

411 is estimated by averaging the time series of velocity components in each

412 grid cell over 10 wave periods after the steady state was reached in each

413 simulation.

414 Bed shear stress

415 Bed shear stress and friction velocity are fundamental variables in sediment

416 transport and scour investigations. Several criteria are employed to

417 determine the initiation of motion, among them the Shields threshold

418 parameter (Shields, 1936) and the so-called wave mobility number

419 (Nielsen, 1992), which can be used to calculate the critical bed shear stress

420 as a function of sediment characteristics. Analysis of spatial variation of bed

421 shear stress, and the mechanisms involved in its increment around the

422 wreck, was conducted. Bed shear stress for the wave conditions and

423 bathymetric profiles was calculated as $\tau=v d u / d z$, where $d z$ is the size of the

424 first grid cell $(\sim 0.005 \mathrm{~m})$.

425 The threshold Shields parameter was calculated according to Soulsby

426 (1997).

$427 \quad \theta_{c r}=\frac{0.3}{1+1.2 D_{*}}+0.055\left[1-\exp \left(-0.02 D_{*}\right)\right] ; \quad D_{*}=d\left[\frac{g\left(\frac{\rho_{S}}{\rho}-1\right)}{v^{2}}\right]^{1 / 3} ;(10)$

428 where $D_{*}$ is the dimensionless grain size, $\mathrm{d}$ is the grain diameter, $v$ is the

429 kinematic viscosity and $\rho_{S}$ is the sediment grain density assumed as quartz

430 density. 
431 The critical bed shear stress follows from the definition of the Shields

432 parameter $\tau_{c r}=d g\left(\rho_{s}-\rho\right) \theta_{c r}$ and is modified for a sloping seabed according

433 to the expressions for downslope flow $\tau_{c}=\tau_{c r}\left[\sin \left(\emptyset_{i}-\beta\right) / \sin \emptyset_{i}\right]$ and upslope

434 flow $\tau_{c}=\tau_{c r}\left[\sin \left(\emptyset_{i}+\beta\right) / \sin \emptyset_{i}\right]$, where $\beta$ is the bed slope and $\emptyset_{i}$ the angle of 435 repose of the sediment $\left(\varnothing_{i}=32 \circ\right.$ according to Soulsby (1997)).

\section{Results}

\section{$437 \quad 4.2$ Oscillatory flow characterization}

438 Figure 3 depicts the vertical distribution of horizontal velocity from the

439 numerical experiment conducted using LEP bathymetry. Velocity profiles

440 correspond to different wave phases as a function of $t^{*}$, being $t^{*}=t / T$. Near

441 bottom velocities around the wreck are increased in the first few

442 centimetres above the seabed. The wreck remains act as a splitter, where

443 the velocity amplification produces fluid separation. As the wave height and 444 period increases, flow velocity increases (Figure $3 a$ and Figure $3 d$ ). The 445 difference between the upwave $(x=191)$ and downwave $(x=198 m)$ velocity 446 profiles are notable. The depth reduction at the offshore $(x<190)$ sediment 447 accumulation increases near bottom orbital velocity, whereas inshore it was 448 reduced $(x<200 \mathrm{~m})$. The differences between upwave and downwave areas 449 become more evident with the increase in wave height and period. Wave asymmetry increases as function of $\mathrm{H}$ and $\mathrm{T}$, especially for larger periods

451 (Figure 3b and Figure 3c). The unperturbed horizontal velocity profile was 452 altered in the nearness of the shipwreck $(x=190-200 \mathrm{~m})$. It is observed 453 upwave and downwave of the wreck (see for example Figure $3 b$ at $t^{*}=6 / 9$ 454 and $7 / 9$ and downwave $(x=198)$ at $t^{*}=2 / 9$ and $\left.t^{*}=4 / 9\right)$. 

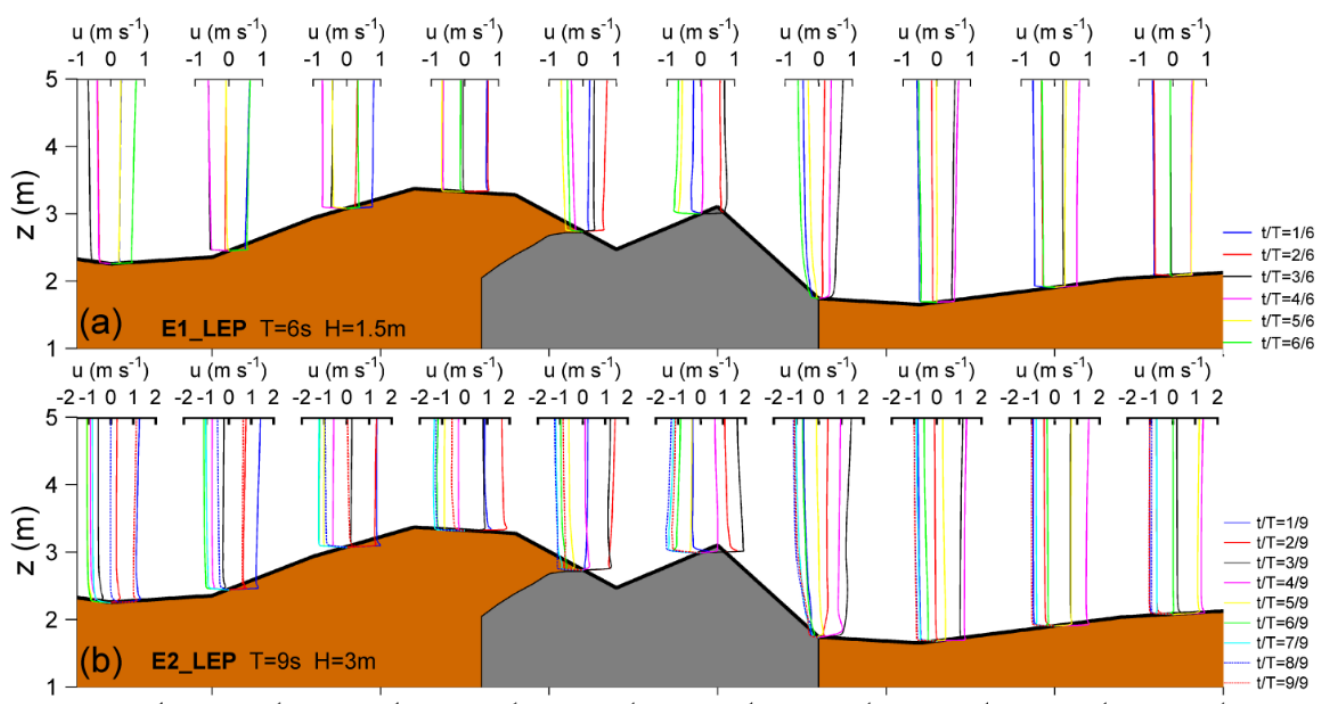

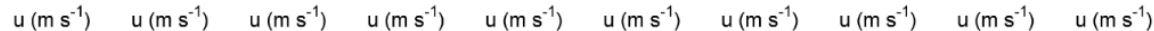

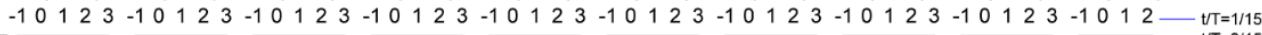

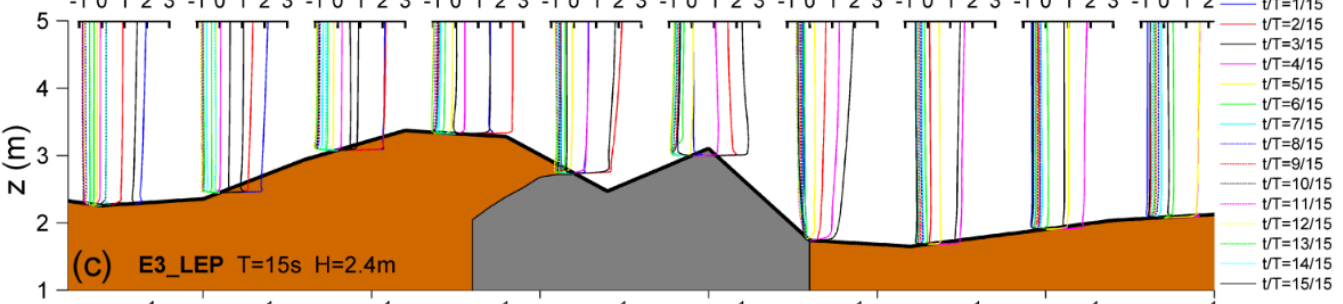

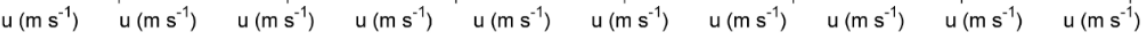

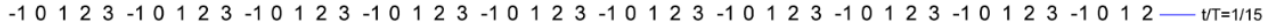

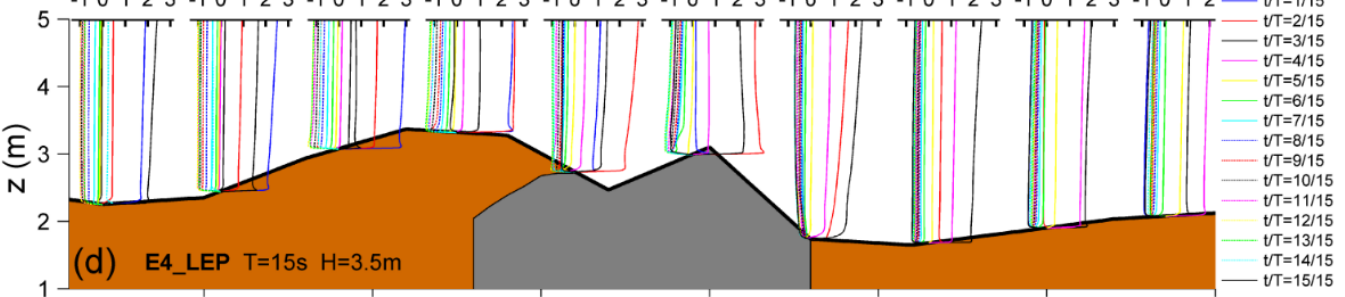

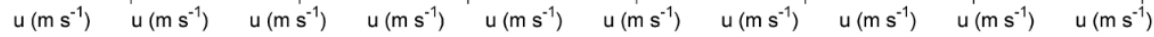

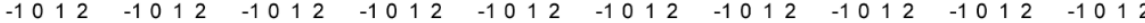

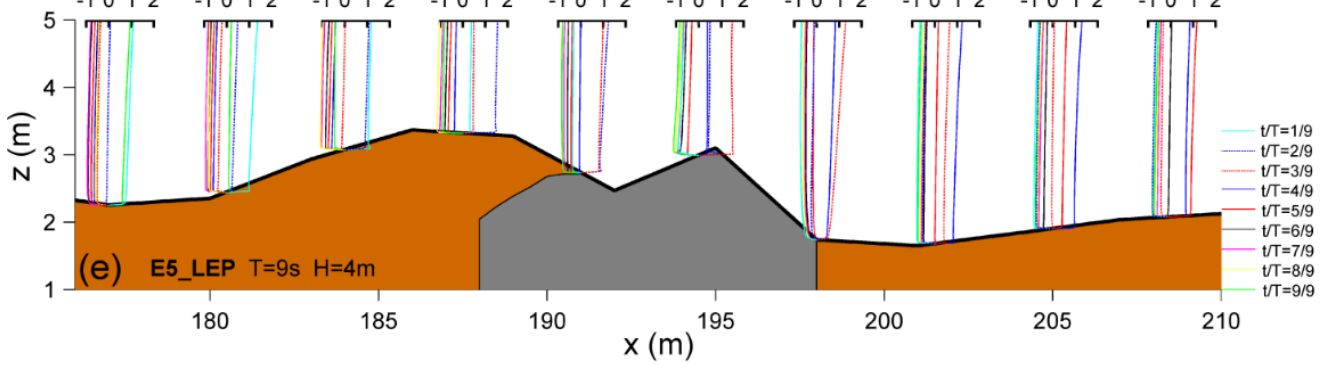

$x(\mathrm{~m})$

456 Figure 3. Vertical distribution of horizontal velocity at different streamwise locations

457 for LEP bathymetry. Information relative to the wave phase variation is represented

458 as a function of the $t^{*}$ ratio for the different line colours and line styles. The grey

colour marks the location of the hull remains.

460 Figure 4 shows the vertical distribution of horizontal velocity for the

461 numerical experiment conducted with the HEP bathymetry. The perturbed 
462 velocity profiles extend further than in the case of LEP ( $x=184 \mathrm{~m}$ and

$463 x=205 m)$, especially downwave of the wreck. A relative increase in

464 maximum near bed velocity is noted with respect to LEP conditions $(x=191 \mathrm{~m}$

465 and $x=194.5 \mathrm{~m}$ ) (Figure $4 \mathrm{a}$ to Figure $4 \mathrm{~d}$ ). Wave height increase and longer

466 periods expand this phenomenon up to the toe of the wreck $(x=198)$

467 (Figure 4d). Despite of depth increase, the inshore scour pit does not

468 produce a notable reduction of near bottom velocity $(x=201.5)$. HEP

469 upwave and downwave dissimilarities are larger than under LEP conditions,

470 with the exception of the offshore wave breaking (Figure 4e). Amplification

471 of wave asymmetry with the increased $\mathrm{H}$ and $\mathrm{T}$ is larger than with LEP at

472 the hull remains (see for example Figure $4 b$ and Figure 4c). The shape of

473 the velocity profiles is markedly modified at the toe of wreck $(x=198 m)$

474 (Figure $4 b\left(t^{*}=4 / 9\right.$ to $\left.\left.t^{*}=7 / 9\right)\right)$, and at the deepest scour pit $(x=201.5)$

475 (Figure $\left.4 b\left(t^{*}=1 / 9,2 / 9,7 / 9,8 / 9,9 / 9\right)\right)$. 

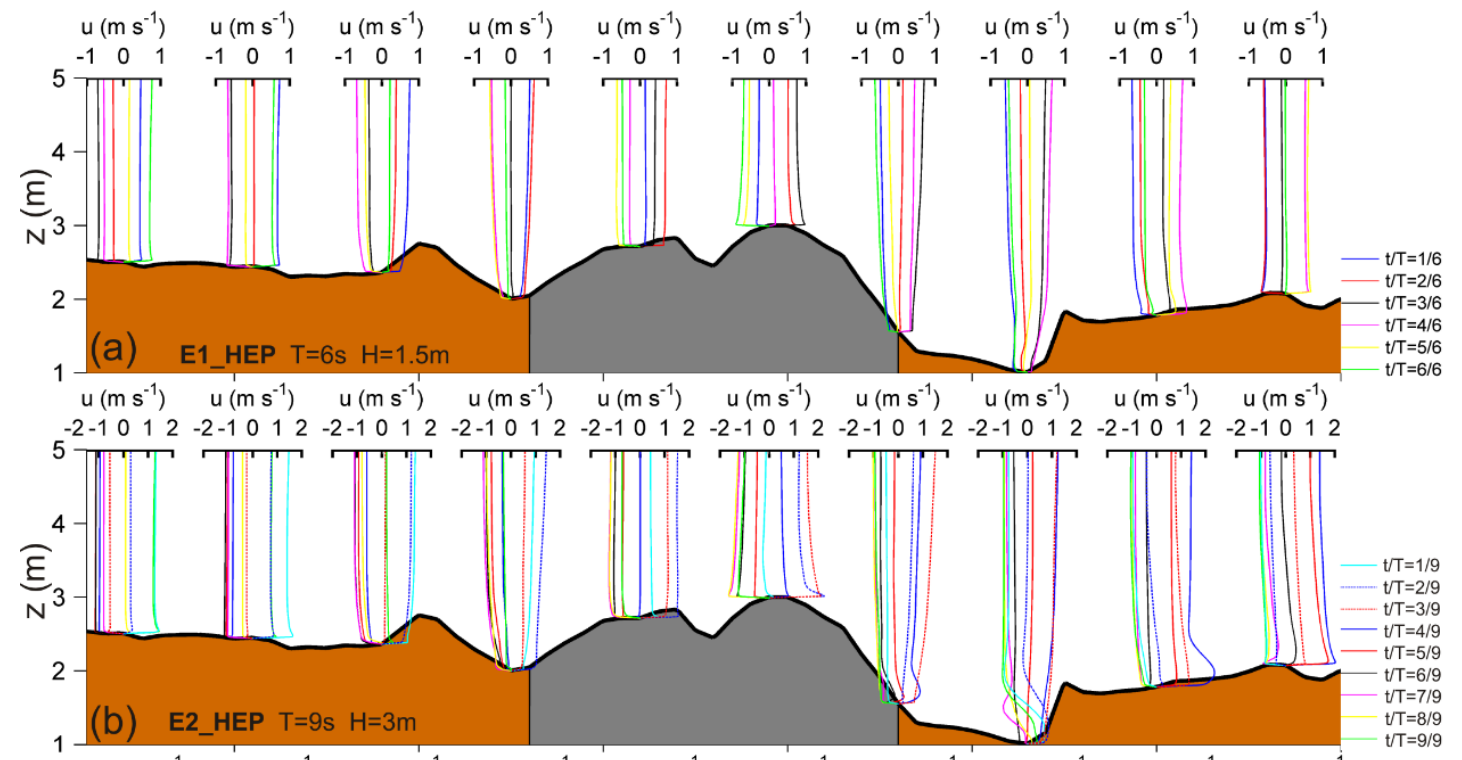

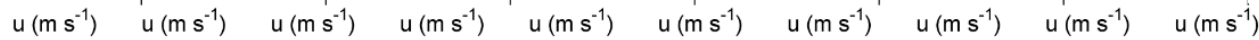

$\begin{array}{rlllllllllllllllllllllllllllllllllllllllllllllllll}-1 & 0 & 1 & 2 & 3 & -1 & 0 & 1 & 2 & 3 & -1 & 0 & 1 & 2 & 3 & -1 & 0 & 1 & 2 & 3 & -1 & 0 & 1 & 2 & 3 & -1 & 0 & 1 & 2 & 3 & -1 & 0 & 1 & 2 & 3 & -1 & 0 & 1 & 2 & 3 & -1 & 0 & 1 & 2 & 3 & -1 & 0 & 1 & 2 & -\end{array}$
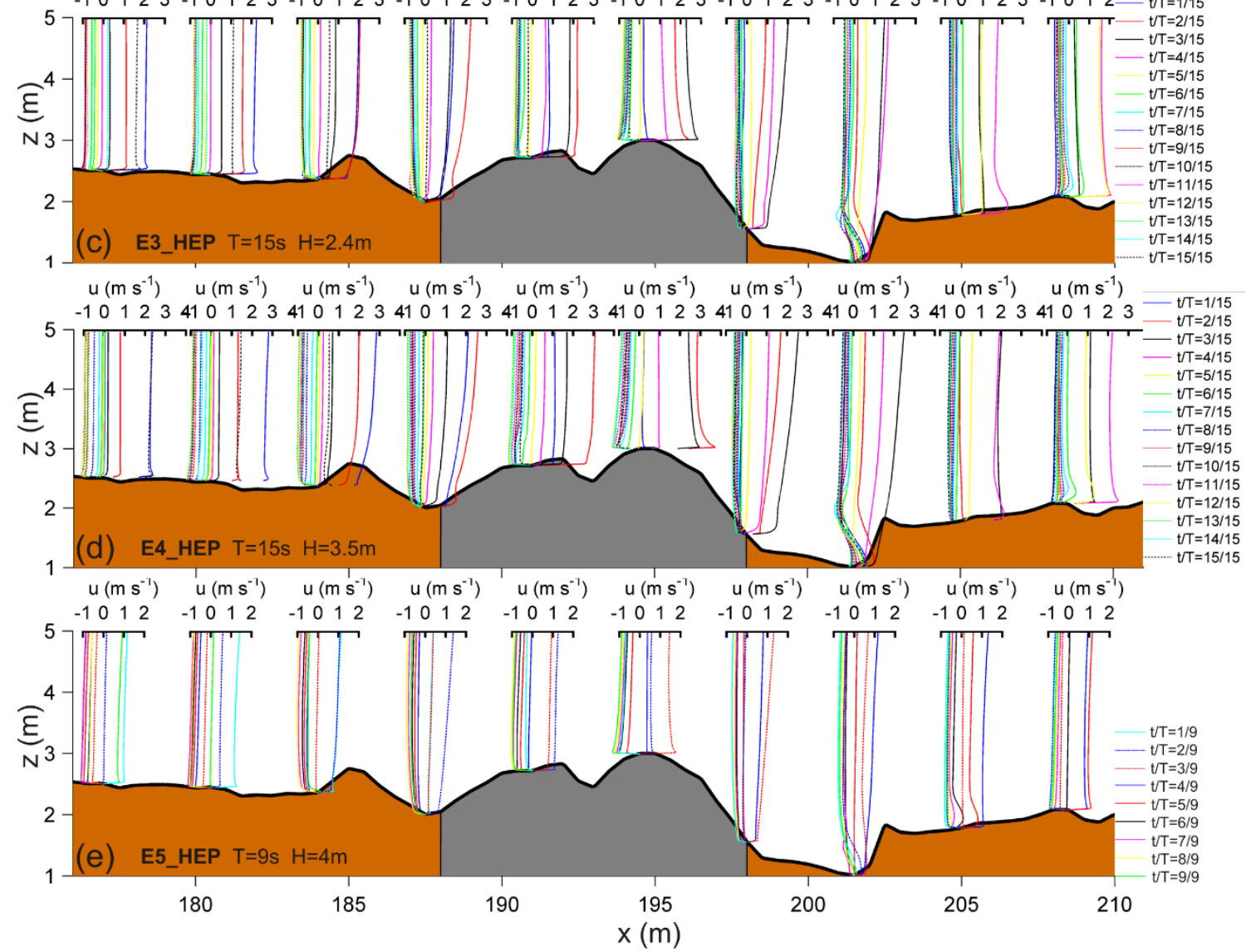

477 Figure 4. Vertical distribution of horizontal velocity at different streamwise locations

478 for HEP bathymetry. Information relative to the wave phase variation is

479 represented as a function of the $t^{*}$ ratio for the different line colours and line styles. 
480 Figure 5 shows the wave Reynolds number $(R e)$ (colour scaled) and

481 boundary layer thickness $(\delta$ ) (black line), under LEP (left panel) and HEP

482 (right panel) conditions. The LEP simulations are dominated by fully

483 turbulent flow, with the exception of the transitional regime observed in

484 E1_LEP (Figure 5a). The wave Reynolds number follows a similar spatial 485 variation in all LEP experiments; amplification over the top of the wreck $486(x=194.5)$ and at the offshore sand accumulation (Figure 5b, Figure 5d). 487 The boundary layer thickness increases from $3.5 \mathrm{~cm}$ in E1_LEP (Figure 5a) 488 to nearly $7 \mathrm{~cm}$ in E4_LEP (Figure $5 \mathrm{~d}$ ). Two peaks are identified at the wreck $489(x=192.4 m)$ and at the toe of wreck remains $(x=197 m)$. In addition, in all 490 experiments $\delta$ increases over the wreck, reaching a maximum between 5 $491 \mathrm{~cm}$ (Figure 5a) and $20 \mathrm{~cm}$ (Figure 5d) at $\mathrm{x} \sim 196 \mathrm{~m}$, which is probably 492 associated with the flow separation point. In the E3_LEP simulation (Figure 493 5c), a maximum is observed upwave of the wreck. In E5_LEP a progressive 494 increase of $\delta$ in the downwave region.

495 The HEP simulations (right panels) also show the predominance of turbulent 496 flow. However, laminar flow at the wreck is observed in E1_HEP (Figure 5f), 497 and a transitional regime in the inshore scour pits and in the offshore scour 498 pits in E2_HEP (Figure 5g). Likewise, a transitional regime was observed at 499 the centre of the wreck site and downwave of the wreck ( $x \sim 210)$ in 500 E5_HEP. The Re increases over the wreck and in the offshore scour crest, 501 decreasing at the inshore and offshore scour pits. The boundary layer 502 thickness shows values slightly higher than those found for LEP experiments 503 and new peaks associated with the offshore scour crest feature ( $x \sim 185)$. 504 However, the larger differences appear downwave of the wreck, at the 505 deepest area of the inshore scour pits (x 200) and especially beyond the 
506 inshore scour pit, where peaks ( $15 \mathrm{~cm}-30 \mathrm{~cm}$ ) are noted (Figure $5 \mathrm{f}$ to

507 Figure $5 j$ ).
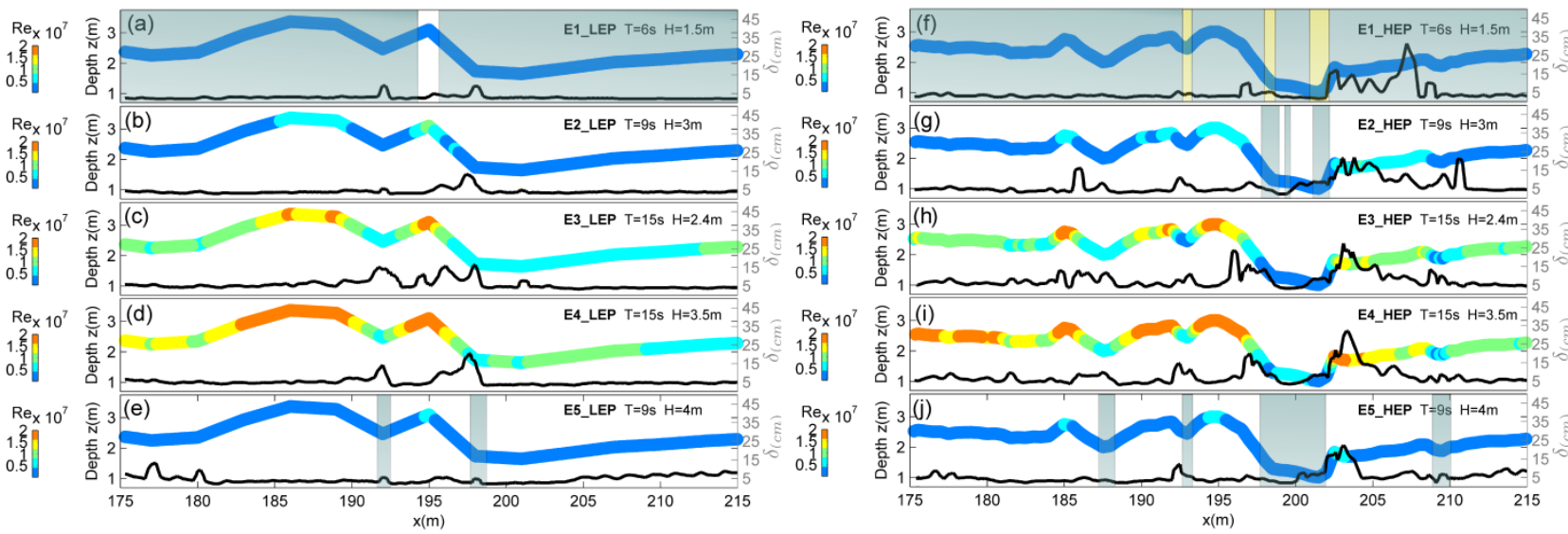

508

509 Figure 5. Wave Reynolds number ( $R e)$ colour scale stamped on bathymetry profile

510 (depth left axis) and boundary layer thickness $(\delta)$ black line (right axis). Left panel

511 shows numerical experiments for LEP conditions. Right panel shows numerical

512 experiment for HEP conditions. The yellow overlay indicates laminar regime

$513\left(\operatorname{Re}<1.5 \cdot 10^{5}\right)$, the grey overlay marks a transitional regime $\left(1.5 \times 10^{5}<R e<10^{6}\right)$, and

514 no colour overlay indicate fully turbulent flow $\left(R e<10^{6}\right)$.

\section{$515 \quad 4.3$ Large coherent structures}

516 The evolution during the wave phase (as a function of $t^{*}=t / T$ ) of

517 dimensionless vorticity $\left(\omega^{*}\right)$ is shown in the left panels, and the

518 corresponding values of the dimensionless Okubo-Weiss parameter $\left(W^{*}\right)$ are

519 shown in the right panels in Figures 6-10. In the case of $\omega^{*}$, the cold colour

520 represents negative vorticity and warm colour represents positive vorticity.

521 In the case of $W^{*}$, the red colour indicates strain dominated areas, the blue

522 colour shows areas dominated by vorticity, and white indicates areas

523 characterized by small positive and negative values of $\mathrm{W}\left(|W| \leq W_{0}\right)$ where

524 the vorticity and strain rate are balanced. Vectors located at position $(x=1$,

$525 Z=195$ ) in the right panels show the sequence of horizontal velocity 
526 registered at $4 \mathrm{~m}$ depth at $\mathrm{x}=194.5$. It should be noted that variable colour

527 scales are used to highlight the different LCS.
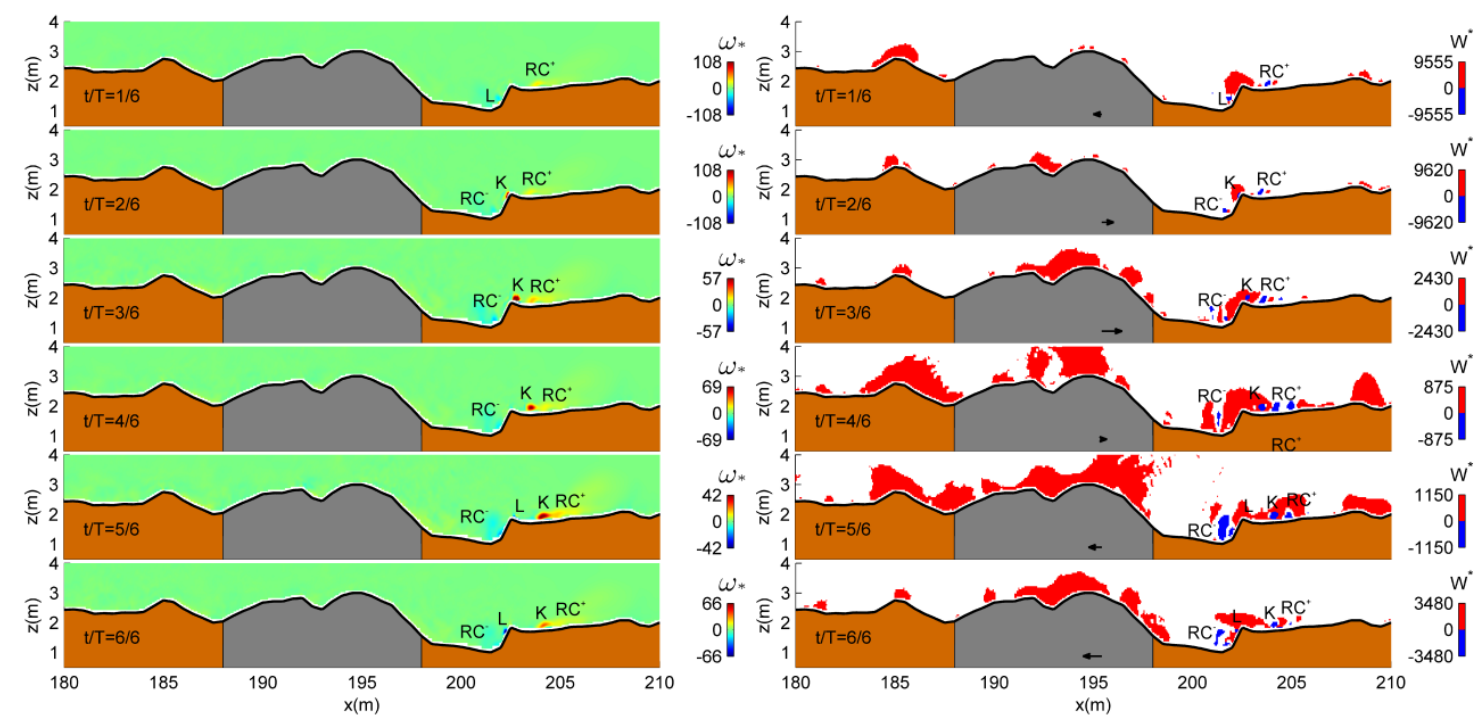

529 Figure 6. Temporal sequence of dimensionless vorticity (left) and the dimensionless

530 Okubo-Weiss parameter (right) for a mean wave cycle in the E1_HEP experiment.

531 Vectors show the horizontal velocity at $4 \mathrm{~m}$ depth at $\mathrm{x}=194.5$.

532 The E1_LEP simulation (not illustrated) does not present relevant vortical

533 structures. On the contrary, in E1_HEP (Figure 6), a vortex $\mathrm{K}$ located at the

534 inshore scour-crest system is flipped over the inshore scour crest $\left(t^{*}=2 / 3\right)$

535 moving shoreward during positive flow acceleration. At the leeside of the

536 inshore scour crest a negative vorticity structure (L) is generated at

$537\left(t^{*}=5 / 6\right)$ flipping over the scour crest during the streamwise negative

538 velocity phase $\left(t^{*}=6 / 6\right)$. A permanent positive vorticity structure $\left(R C^{+}\right)$is

539 located downwave and $\mathrm{RC}^{-}$is found at the inshore scour pits and is present

540 during all wave phases.

541 In simulation E2_LEP (Figure 7) a counterclockwise rotation vortex (positive

542 vorticity) originates upwave and flips over the wreck $\left(t^{*}=2 / 6\right)$ to reach the

543 toe of hull remains at $\mathrm{t}^{*}=4 / 9$. On the offshore side, a small $\mathrm{K}^{\prime}$ vortex $\left(\omega^{*}>\right.$ 
$5440)$ formed above the sediment accumulation moves towards the wreck $\left(t^{*}=\right.$

$5452 / 9-5 / 9)$ with shoreward flow and in the opposite direction ( $\left.t^{*}=6 / 9-8 / 9\right)$.

546 Another small vortex $L^{\prime}\left(\omega^{*}<0\right)$ produced upwave of the wreck $\left(t^{*}=2 / 9\right)$

547 moves around the hull $\left(t^{*}=5 / 9-9 / 9\right)$. A small eddy $L$ with negative vorticity

548 generated $\left(t^{*}=2 / 9\right)$ at the toe of the wreck is dragged shoreward during the

549 positive horizontal velocity phase $\left(t^{*}=4 / 9-5 / 9\right)$. In addition, the location of

550 a larger counter-clockwise rotation structure $\left(\mathrm{RC}^{+}\right)$oscillates upwave-

551 downwave with the horizontal velocity throughout the whole wave cycle.
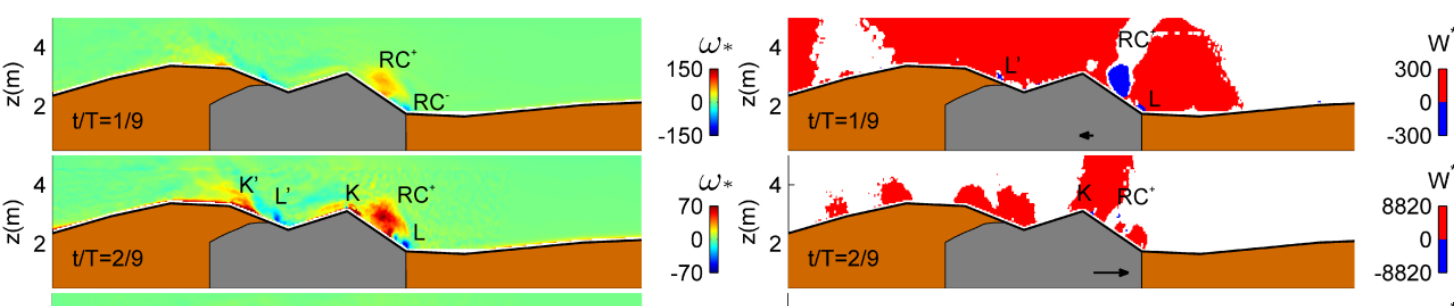

$W$
8820
0
8820
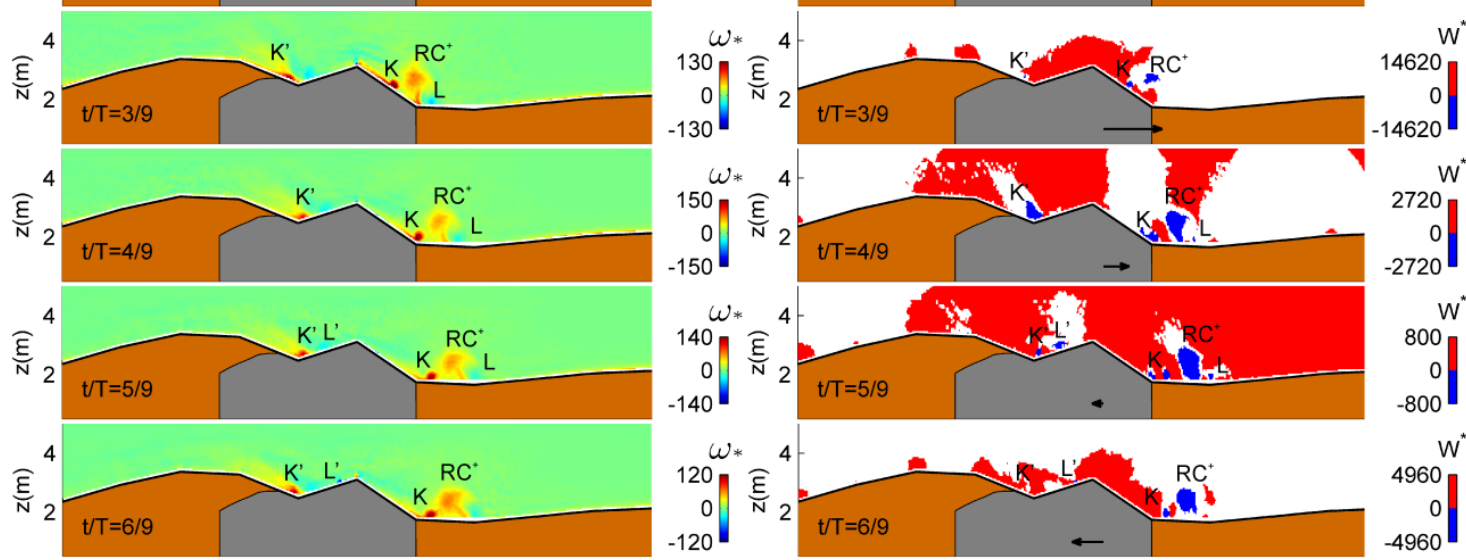

$W^{*}$
2720
0
2720
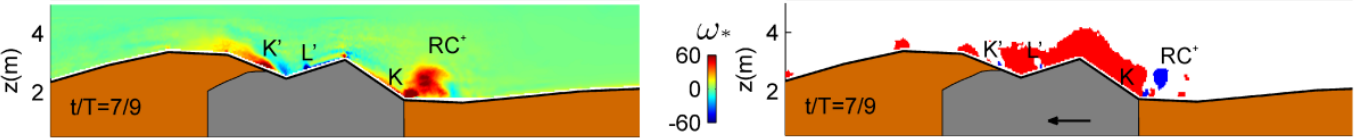

$W^{*}$
800
0
-800
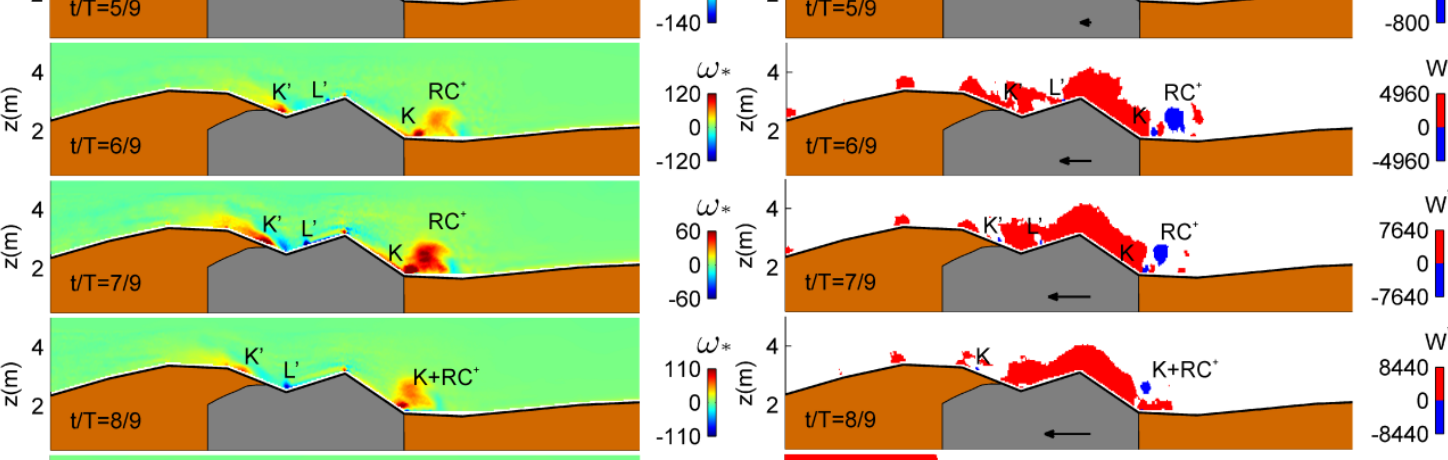

$W^{*}$
4960
0
-4960
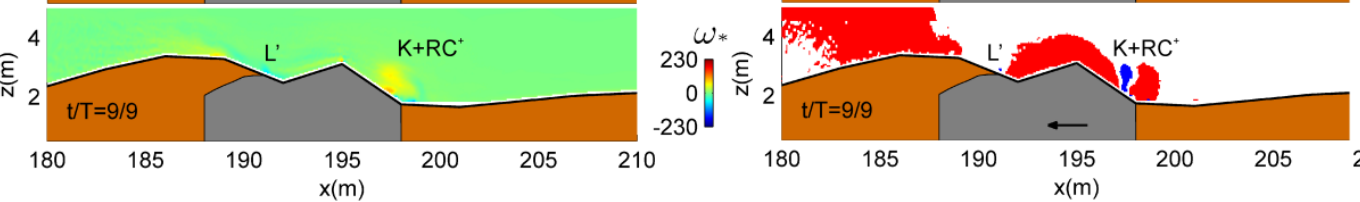

W
7640
0
7640

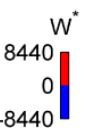

Figure 7. Temporal sequence of dimensionless vorticity (left) and the dimensionless

554 Okubo-Weiss parameter (right) for a mean wave cycle in the E2_LEP experiment.

555 Vectors show the variation of horizontal velocity at $4 \mathrm{~m}$ depth at $\mathrm{x}=194.5$. 
556 The results corresponding to the simulation E2_HEP are shown in Figure 8.

557 An eddy with positive vorticity $\mathrm{K}^{\prime}$ generated in the previous wave cycle over

558 the wreck $(x=193)$ is washed over the hull remains when the wave

559 propagates and it reaches the toe of the wreck $\left(t^{*}=4 / 9-5 / 9\right)$ (right panels

560 Figure 8). Subsequently, during the negative horizontal velocity phase, $\mathrm{K}^{\prime}$ is

561 carried seawards over the wreck from $t^{*}=5 / 9$ to $t^{*}=9 / 9$, similar to

562 E2_LEP. The flow acceleration produces elongation of the vortex in the

563 horizontal direction.

564 At the inshore scour pit, a larger $\mathrm{K}$ vortex with counterclockwise rotation is

565 observed at $t^{*}=1 / 9$, along with the vortex $\left(K_{0}\right)$ developed during the

566 previous wave cycle. $K$ is generated by seaward flow( $\left.t^{*}=6 / 9\right)$. It flips over

567 the inshore scour crest during a positive horizontal velocity phase, and

568 forms a paired vortex with a small clockwise vortex (L) formed at the top of

569 scour crest $\left(t^{*}=2 / 9\right)$. They subsequently shed, moving seaward $\left(t^{*}=2 / 9\right.$ -

$\left.570 t^{*}=5 / 9\right)$ as is shown by the vorticity field. At the moment of maximum

571 positive velocity $\left(t^{*}=3 / 9\right)$, after the flow acceleration phase, a new

572 clockwise vortex (M) arises at the lee side of the scour crest $(x=202.5)$. The

573 M vortex initially forms a vortex pair with $K_{2}$ and it is washed seaward as far

574 as the toe of the wreck. At the offshore scour pit, a small vortex $K^{\prime \prime}\left(\omega^{*}>0\right)$

575 is observed trapped between $x=186$ and $x=188$. 

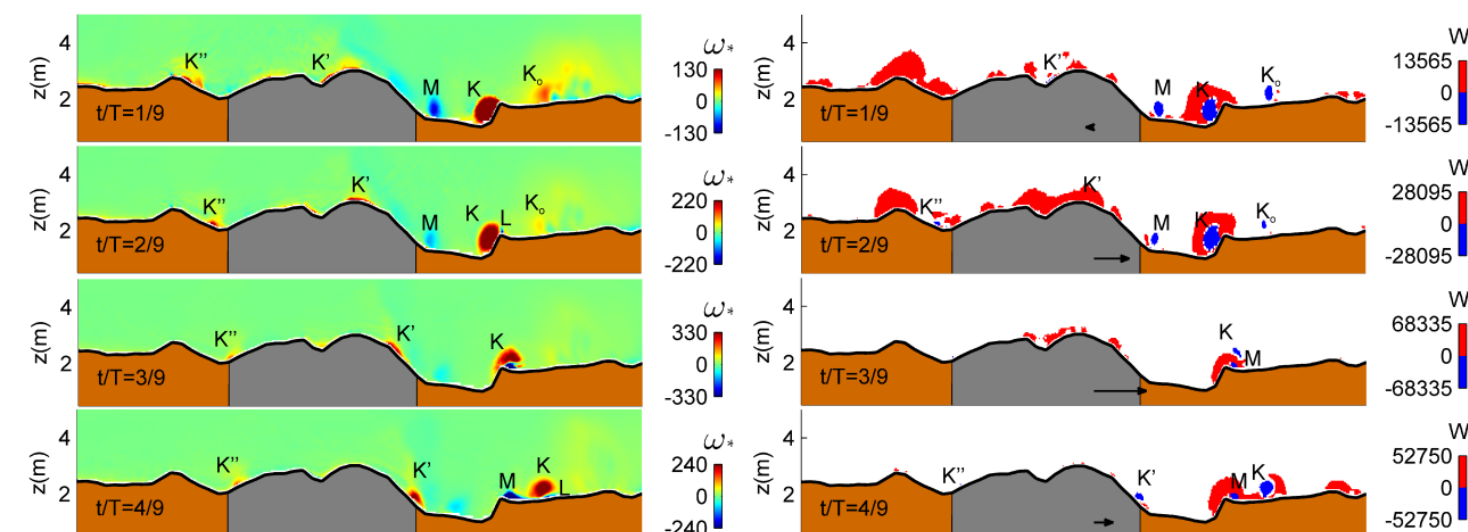

W

$\omega_{*}$
240
0
-240

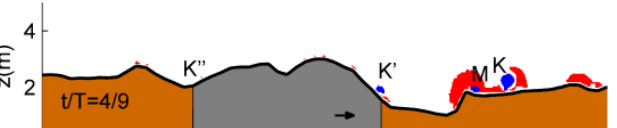

0
-68335

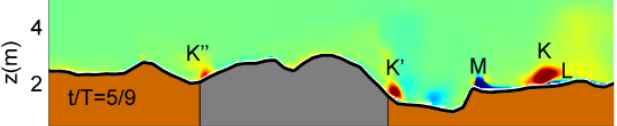

$\omega_{*} \omega_{*}$
0
-150

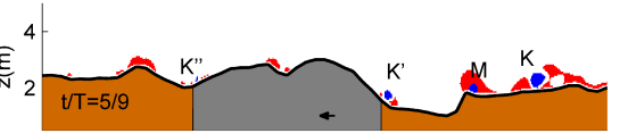

W

2750
0

$-52750$

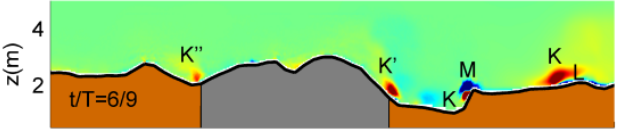

160
0
-160

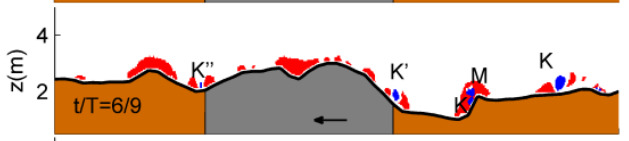

W
8435
0

$-18435$

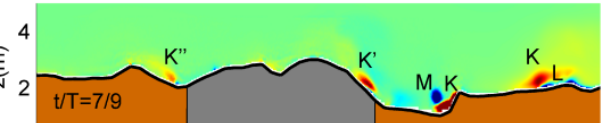

${ }_{170}^{\omega_{*}}$

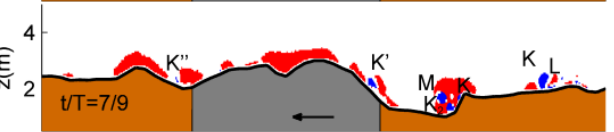

16635
0

W

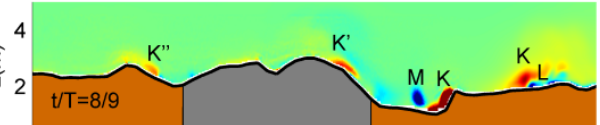

$\omega_{*}$
140
0
-140

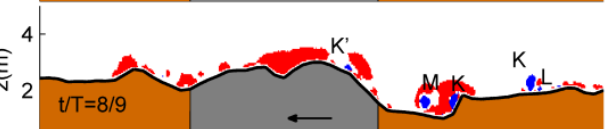

0
-20565
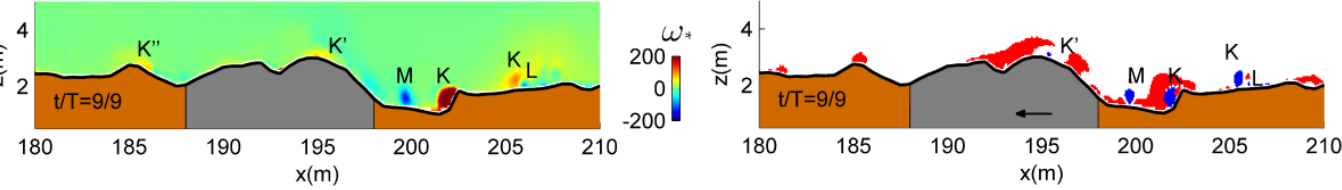

W"

0

W

577 Figure 8. Temporal sequence of dimensionless vorticity (left) and the dimensionless

578 Okubo-Weiss parameter (right) for a mean wave cycle in the E2_HEP experiment.

579 Vector show the variation of horizontal velocity at $4 \mathrm{~m}$ depth at $\mathrm{x}=194.5$

580 The vortex dynamics corresponding to simulation E3_LEP (not shown) is

581 similar to E2_LEP without the development of the $\mathrm{RC}^{+}$and $\mathrm{L}$.

582 In the E3_HEP (Figure 9) vortex dynamics is similar to that of E2_HEP at

583 the inshore scour-crest. However, unlike E2_HEP, the M vortex crosses the

584 inshore scour pits detached from the seabed, and rises when it reaches the

585 wreck. Subsequently, at the beginning of the wave cycle $\left(t^{*}=1 / 15\right), M_{\circ}$ (the

586 vortex $M$ in the previous wave cycle) is transported shoreward (left panel at

$587 t^{*}=7 / 15$, and right panel at $\left.t^{*}=8 / 15\right)$. On the other hand, it should be 
588 noted that the large $L^{\prime}$ vortex $\left(\omega^{*}<0\right)$ generated downwave of the wreck

589 during the positive horizontal velocity phase, flips over the wreck $\left(t^{*}=6 / 15\right.$ -

590 14/15). Also, a L" vortex (negative vorticity) located upwave of hull remains

591 originates at the the wreck site $(x=192.7 \mathrm{~m})$ at $t^{*}=4 / 15$ and is transported

592 by the mean flow seaward to the offshore scour crest $\left(t^{*}=5 / 15-5 / 15\right)$.

593 At the offshore scour-crest system, the $\mathrm{K}^{\prime \prime}$ vortex is identified only at

$594 t^{*}=1 / 15-2 / 15$ at the leeside of scour-crest. Likewise, a vortex $L^{\prime \prime \prime}$ is also

595 captured when it flips over the scour-crest $\left(t^{*}=7 / 15-12 / 15\right)$. 

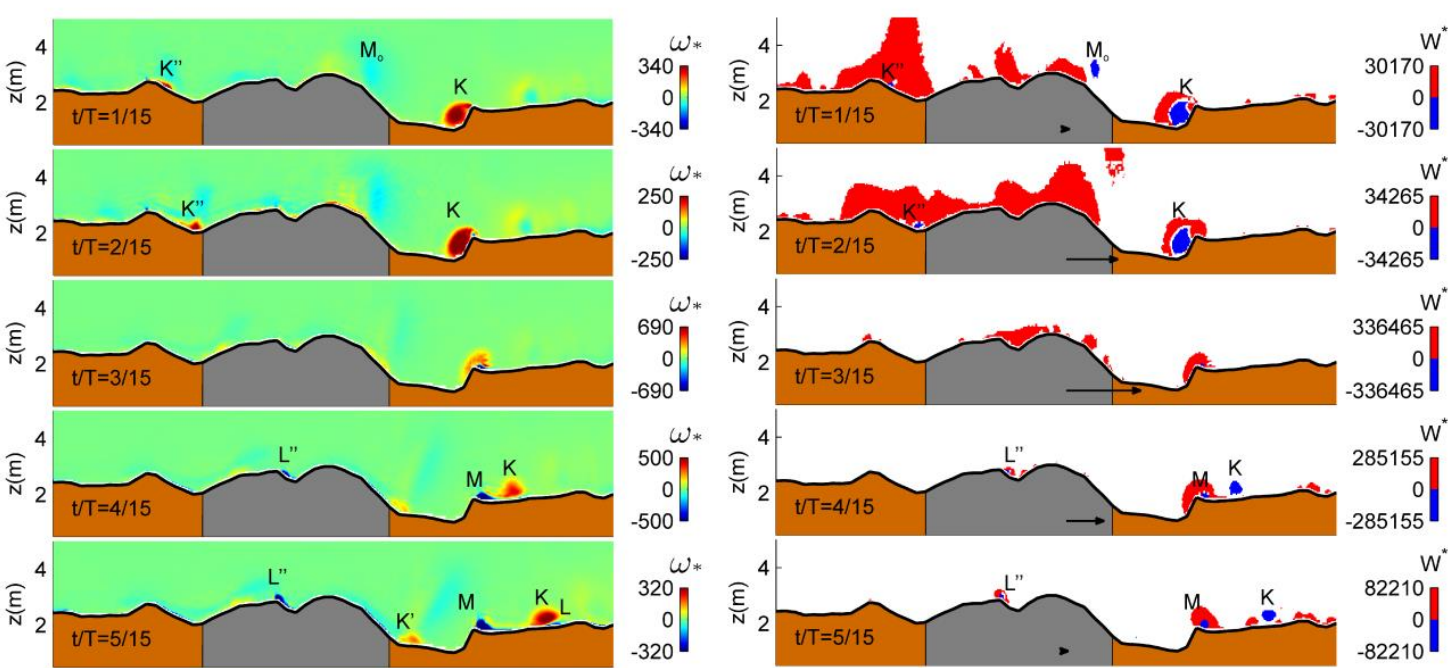

$W^{*}$
82210
0
-82210

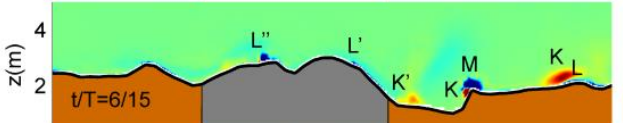

$\omega_{*}$
350
0
-350

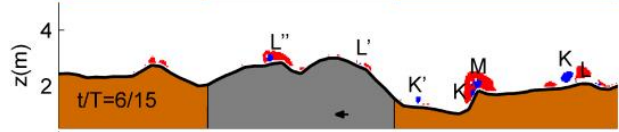

$W^{*}$
40310
0
-40310

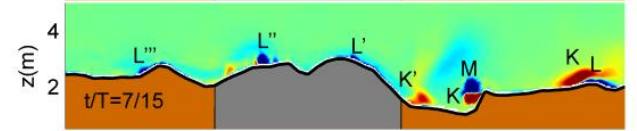

$\omega_{*}$
150
0
-150
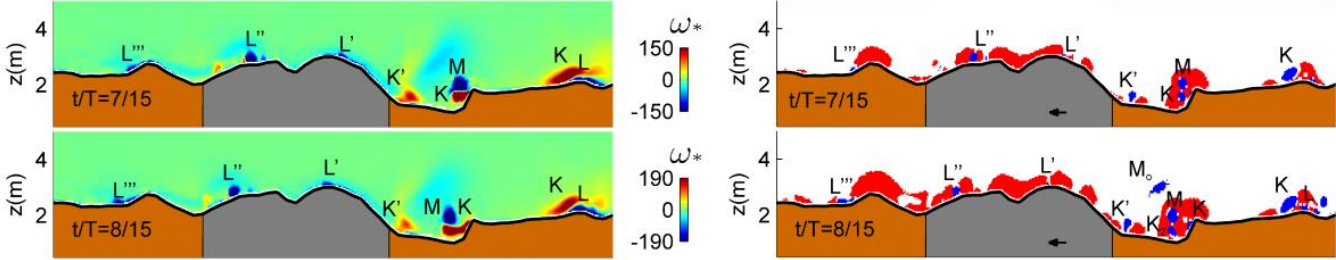

$\omega_{*}$
190
0
-190

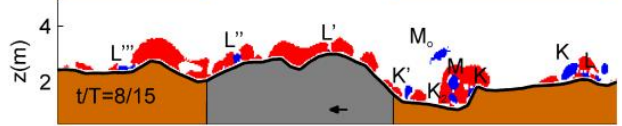

$W^{*}$
9540
0
-9540

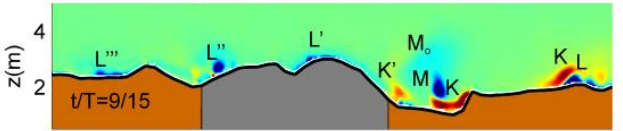

$\omega_{*}$
190
-190

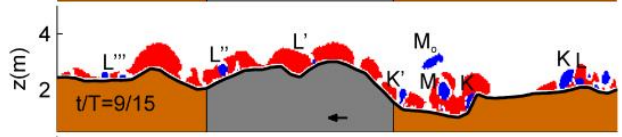

$W^{*}$
8780
0
-8780

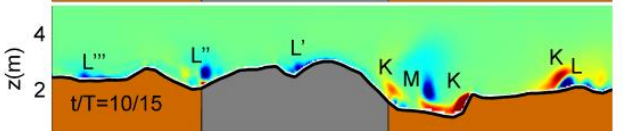

$\omega_{*}$
0
0
-180
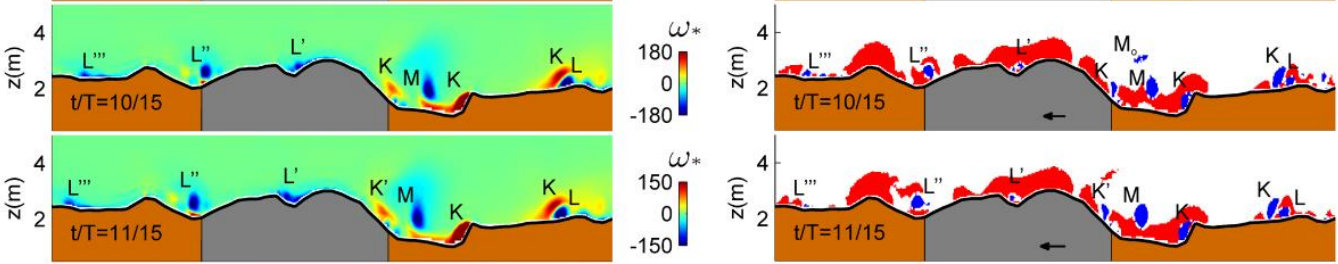

$W^{*}$
5415
0
-5415

$-5415$

$\omega_{*}$
150
0
-150
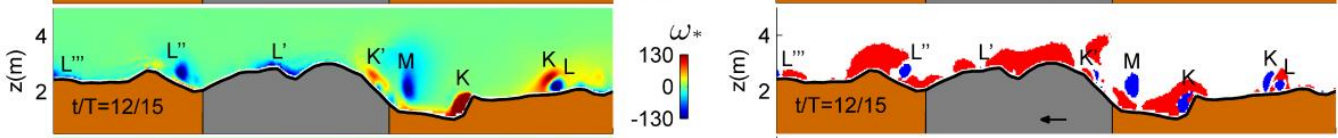

W
5590
0
-5590

$-5590$

$W^{*}$
7790
0
-7790

$\omega_{*}{ }^{130}$
0
-130

$-7790$

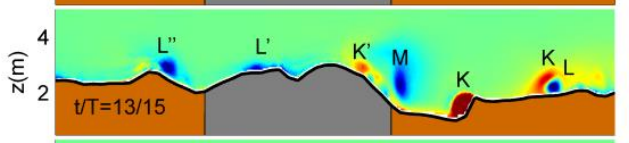

${ }_{120}^{\omega_{*}}{ }^{-120}$

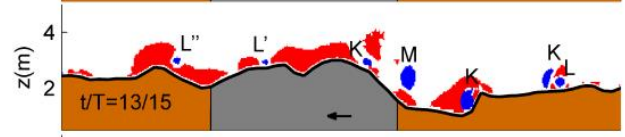

$W^{*}$
11670
0
-11670

$-11670$

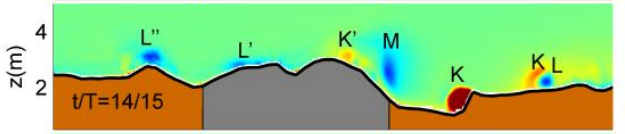

160
0
0
-160
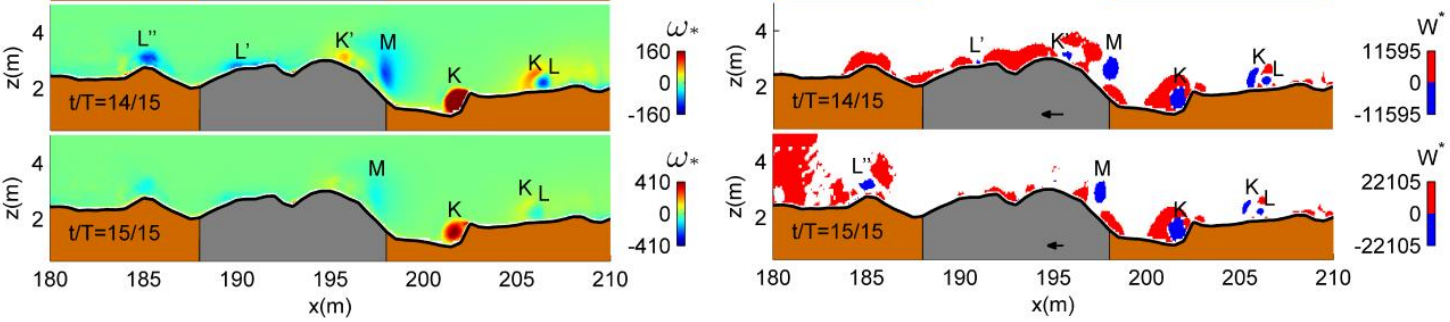

597 Figure 9. Temporal sequence of dimensionless vorticity (left) and the dimensionless

598 Okubo-Weiss parameter (right) for a mean wave cycle in the E3_HEP experiment.

599 Vector show the variation of horizontal velocity at $4 \mathrm{~m}$ depth at $\mathrm{x}=194.5$ 
600 E4_LEP vorticity pattern resembles that of E3_LEP, although some

601 differences appear. The eddy with counterclockwise rotation at the toe of

602 the wreck is horizontally elongated, and is eventually split into two vortices

$603\left(t^{*}=6 / 15\right)$ by the mean flow. Additionally, there is an increase in size in the 604 clockwise vortex at the centre of the wreck site $\left(t^{*}=10 / 15\right)$. The $L^{\prime \prime}$ vortex 605 generated at the centre of the wreck site during the deceleration phase of shoreward flow is strained over the sediment accumulation $(x=185)$ during

607 the seaward flow phase.

608 The system of coherent structures developed in simulation E4_HEP (Figure 609 10) shows higher complexity than in the other cases. At the inshore scour610 crest system, the process of vortex formation and shedding is similar to the 611 E3_HEP experiment. A larger K-type vortex of $1.2 \mathrm{~m}$ diameter is located at 612 the inshore scour mark, and an M vortex is generated after the maximum 613 positive velocity downwave of scour crest. In this case, an $\mathrm{L}$ and $\mathrm{K}$ vortex 614 pair emerge $\left(t^{*}=5 / 15\right)$ at $x \sim 208$. Additionally, a system of small vortices is 615 present at $x=208-210$.

616 In the inshore scour pits the $K$ vortex $\left(t^{*}=7 / 15\right)$ is also divided generating a $617 \mathrm{~K}_{2}$ vortex. The negative vorticity eddy $M$ created downwave of the inshore 618 scour ridge $(x=202)$ at $t^{*}=3 / 15$, is larger in size $(1.10 \mathrm{~m}$ diameter $)$ and it 619 follows a seaward trajectory detached from the seabed.

620 At the wreck, the vortex formation process is better developed than in 621 previous simulation and is accompanied by a new clockwise eddy $\mathrm{N}$ $622\left(t^{*}=7 / 15-9 / 15\right)$. The $L^{\prime}$ vortex splits into smaller vortices as the seaward 623 flow washes over the hull structure $\left(t^{*}=7 / 15-9 / 15\right)$. Likewise, the $L^{\prime \prime}$ vortex 624 formed in the centre of the wreck site divides into smaller eddies. 
625 At the offshore scour crest a similar process takes place, where vortex $L^{\prime \prime \prime}$

626 flips over the offshore crest and divides into smaller structures during the 627 negative velocity phase $\left(t^{*}=10 / 15\right)$ 

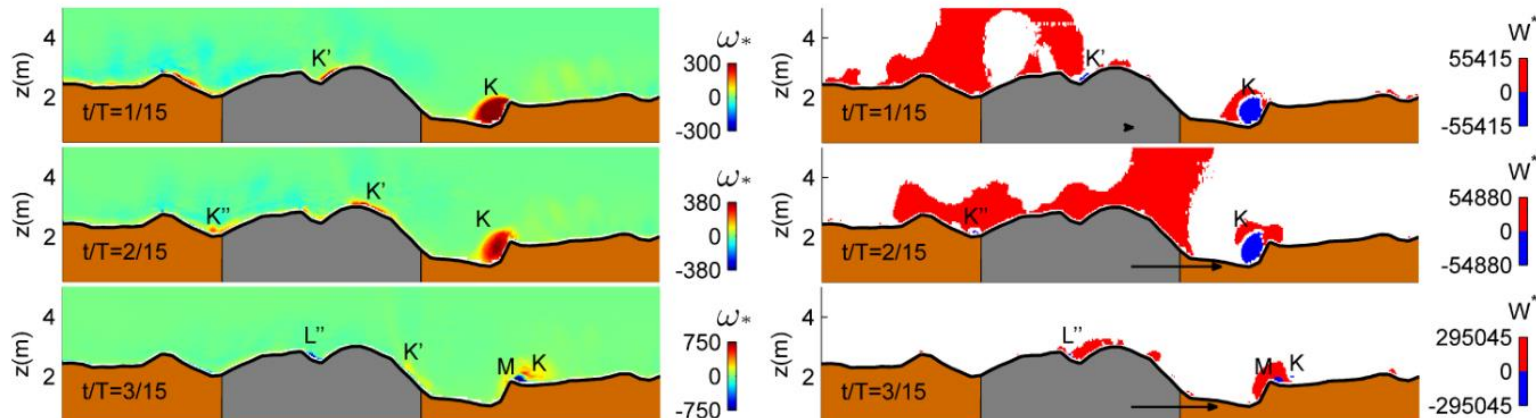

W

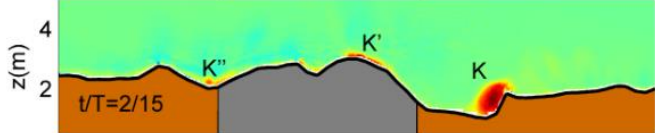

295045
0
-295045
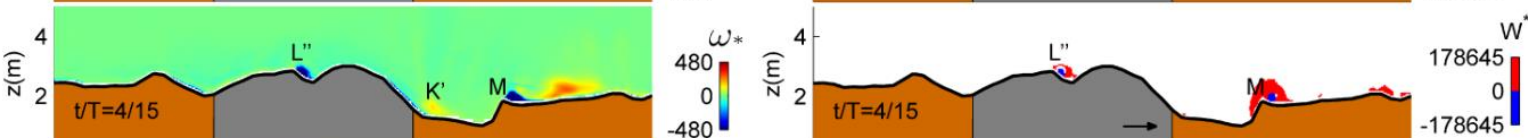

178645
0
-178645
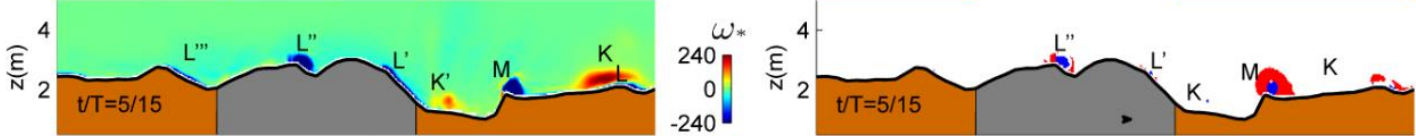

W
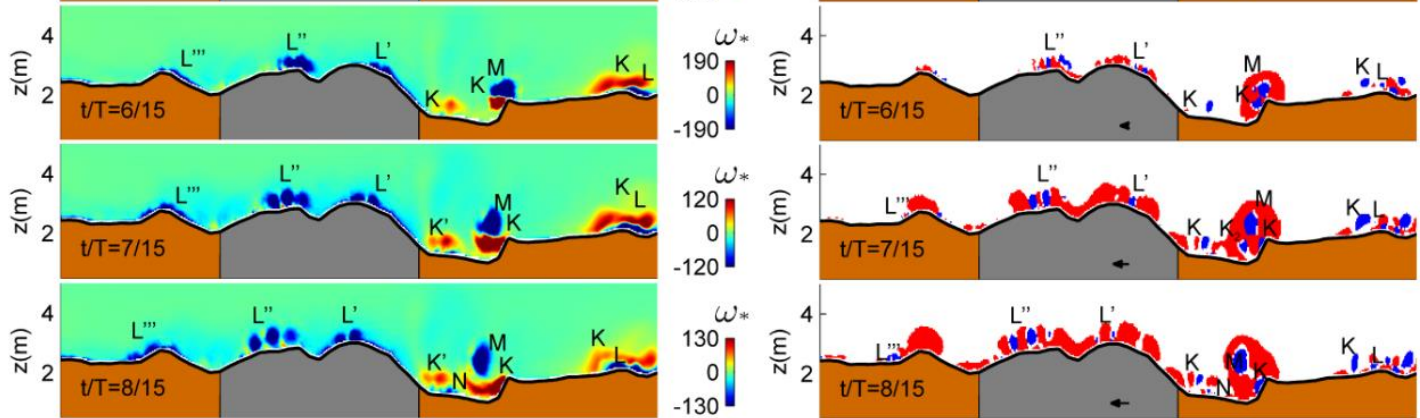

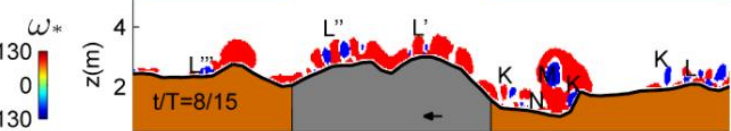

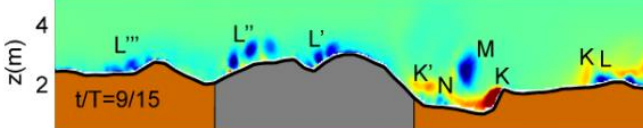

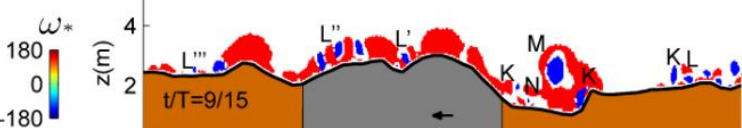
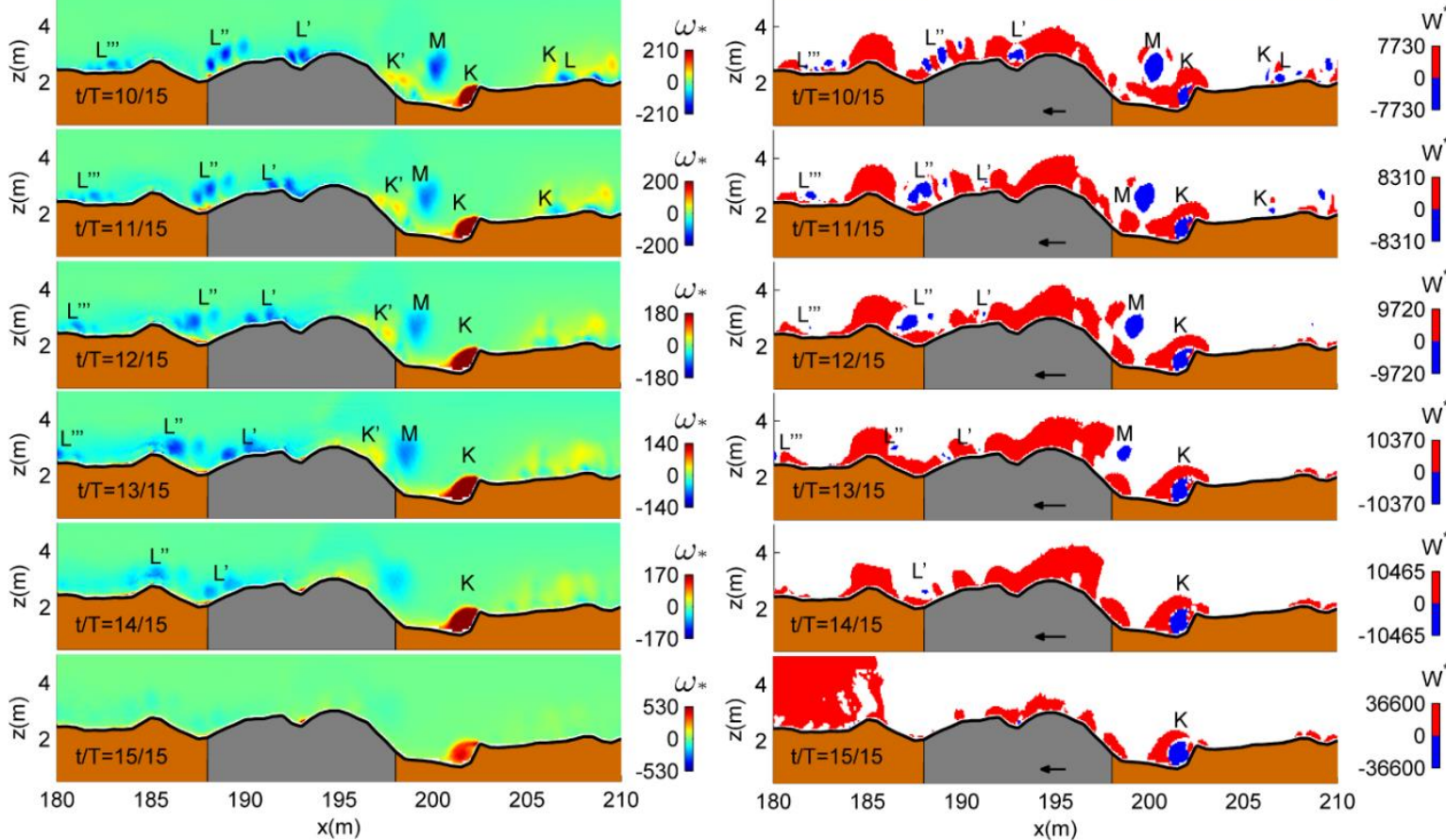

$W$
36600
0
-36600

629 Figure 10. Temporal sequence of dimensionless vorticity (left) and the

630 dimensionless Okubo-Weiss parameter (right) for a mean wave cycle in the E4_HEP 
631 experiment. Vector show the variation of horizontal velocity at $4 \mathrm{~m}$ depth at

$632 x=194.5$

633 Simulation E5_LEP (not shown) is similar to E2_LEP. In E5_HEP, vortices

634 are only identified in the inshore and offshore scour-crest systems. At the

635 inshore, the two vortex pairs upwave and downwave of the scour crest are

636 observed simultaneously, remaining on or near the scour crest, unlike in

637 previous HEP simulations. Also, the $L$ vortex of the $K-L$ pair downwave of

638 the scour crest grows in size $\left(t^{*}=7 / 9-8-9\right)$.

\section{$639 \quad 4.4$ Turbulence}

640 Figure 11 shows the vertical distribution of turbulent shear stress from

641 simulations using LEP bathymetry with maximum values located around the

642 wreck $(x=190-200)$. Shear stress profiles located at the offshore sediment

643 accumulation show a higher level of turbulence near bed in E3_LEP (Figure

644 11c), E4_LEP (Figure 11d) and E5_LEP (Figure 11e). In the case of E4_LEP,

645 after the wave breaking point $(x=200)$ no increment of turbulent shear

646 stress at the seabed is observed. However, in E5_LEP (wave breaking point

647 at $x=89$ ) higher levels of turbulence are observed on the offshore side of

648 the wreck. 

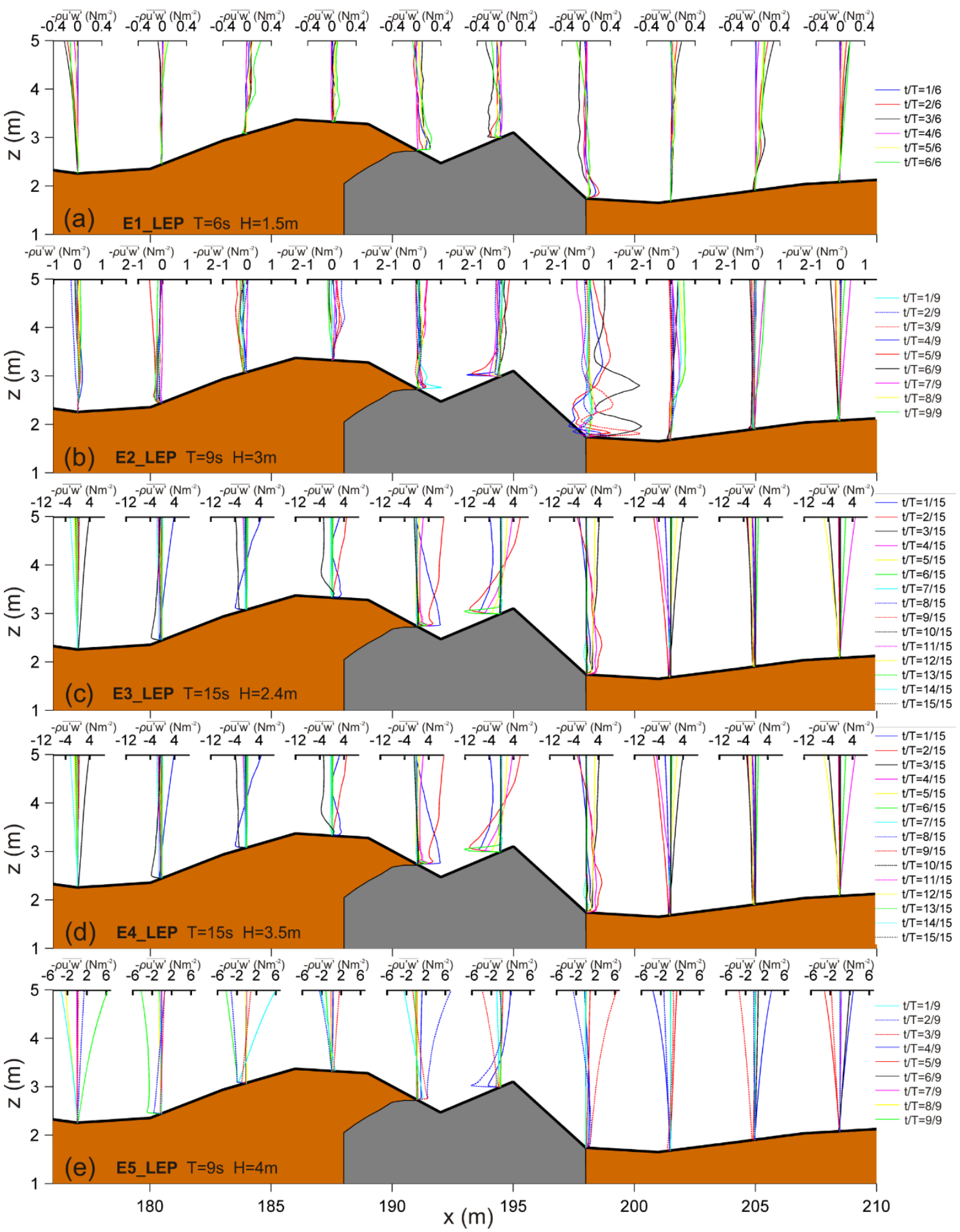

Figure 11. Vertical distribution of residual horizontal turbulent shear stress

$651\left(-\rho \overline{u^{\prime} w^{\prime}}\right)$ at different streamwise locations simulated with the LEP

652 bathymetry (note that different scales are used in the experiments to

653 highlight the variation of $-\rho \overline{\mathrm{u}^{\prime} \mathrm{w}^{\prime}}$ ). Information relative to the wave phase 
654 variation is represented as a function of the $t^{*}$ ratio for the different line 655 colours and styles

656

657 In the case of HEP bathymetry (Figure 12) the areas of maximum shear 658 stress are concentrated downwave of the wreck, in the near bed region. 659 Unlike the LEP simulation, near bed shear stress is reduced upwave of the 660 hull remains with respect to the downwave area. Similar to the LEP 661 simulation, wave breaking introduces higher levels of turbulence into the 662 system as indicated by larger values of shear stress (Figure 12d). 


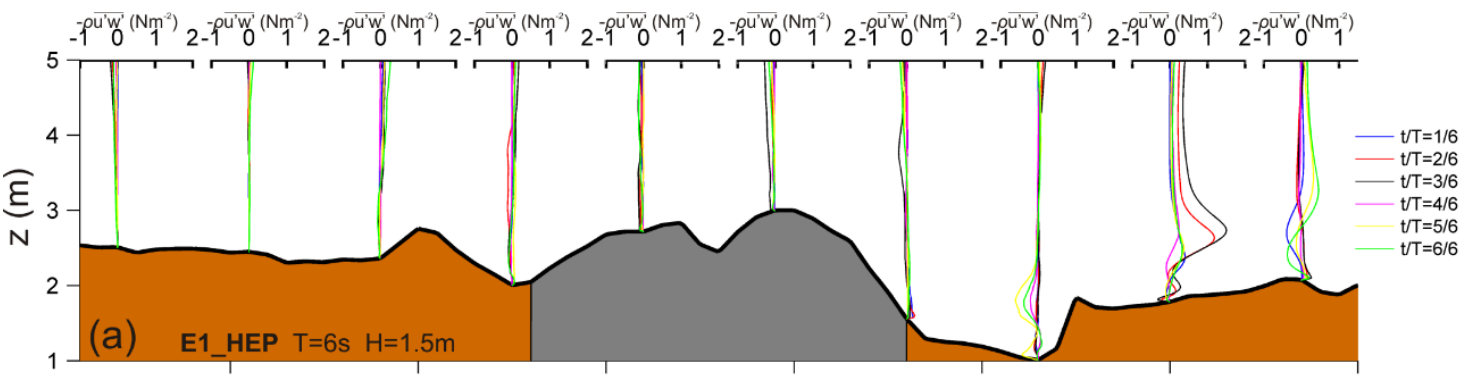

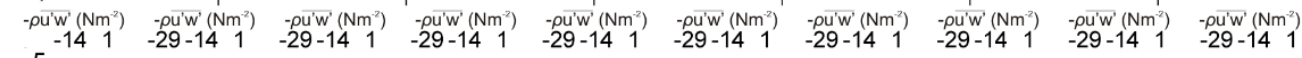
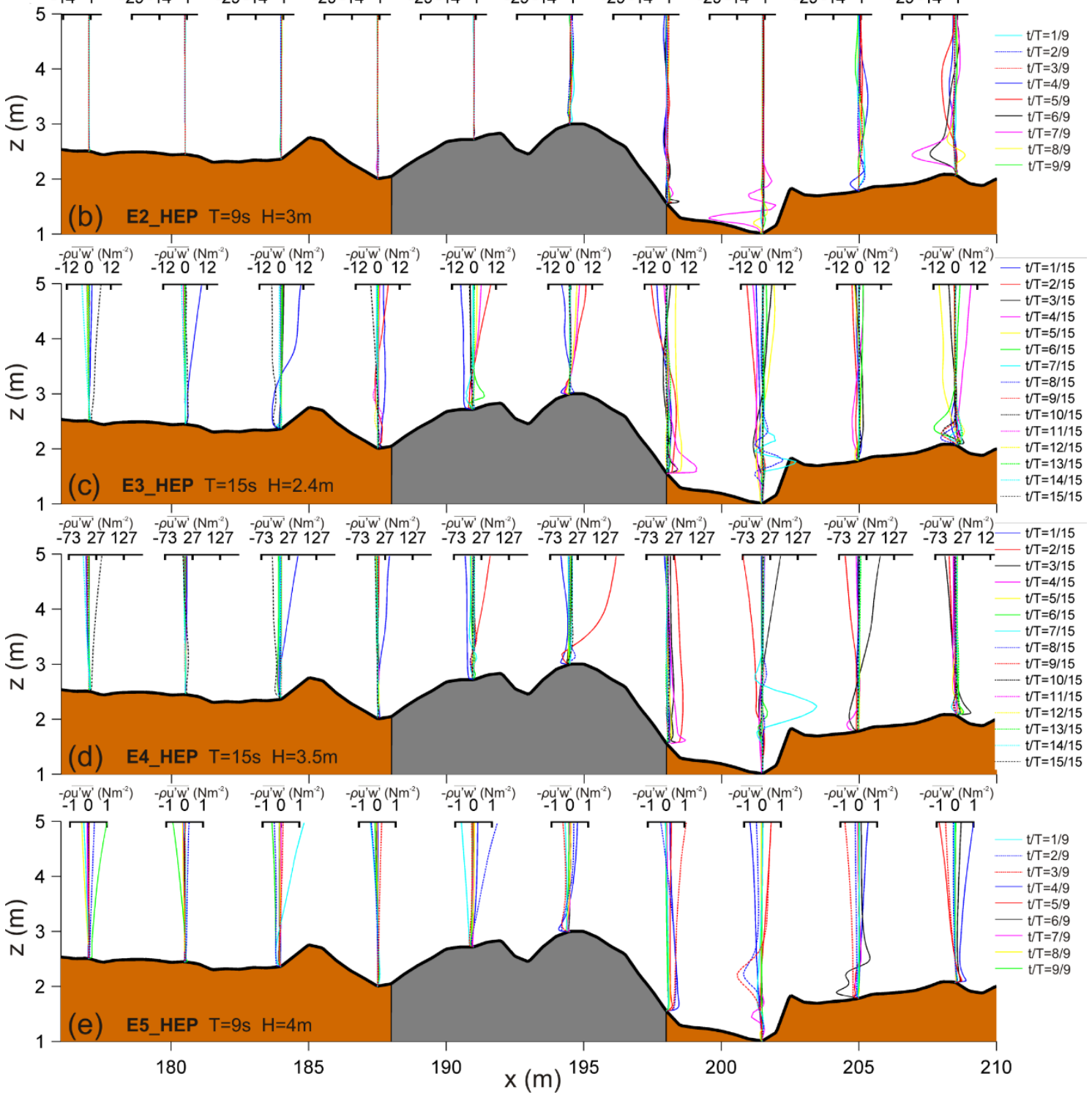

664 Figure 12. Vertical distribution of residual horizontal turbulent shear stress $\left(-\rho \overline{\mathrm{u}^{\prime} \mathrm{w}^{\prime}}\right)$

665 at different streamwise locations simulated with the HEP bathymetry (note that

666 different scales are used in the experiments to highlight the variation of $-\rho \overline{\mathrm{u}^{\prime} \mathrm{w}^{\prime}}$ ).

667 Information relative to the wave phase variation is represented as a function of $\mathrm{t}^{*}$

668 ratio for the different line colours and line stile. 


\subsection{Steady currents generated by nonlinear effects induced by}

670 waves (SCw): undertow and steady streaming.

671 LEP simulations indicate steady currents (Figure 13, left panels) are more

672 intense in the near bed region over the hull remains and the offshore

673 sediment accumulation. The increment in wave height and periods produces

674 SCw intensification (see Figure 13a and Figure 13c). A counterclockwise

675 current cell developed at the toe of the hull (x 197m) and the SCW

676 intensification is observed in the near bottom region in E1_LEP(Figure 13a).

677 In the case of simulation E3_LEP (Figure 13e) the SCw enhancement $(<0.3$

$678 \mathrm{~m} \cdot \mathrm{s}^{-1}$ ) is mainly restricted to the near bed region. In E4_LEP, wave breaking

679 produces an increase in SCw $\left(0.3 \mathrm{~m} \cdot \mathrm{s}^{-1}\right)$ over the wreck, at the offshore sand 680 accumulation (from $x=180$ to $x=190$ ) and downwave of the breaking point $681(\mathrm{x} \sim 200 \mathrm{~m})$. Larger SCw values $\left(0.4 \mathrm{~m} \cdot \mathrm{s}^{-1}\right)$, especially upwave of the wreck, 682 are simulated in the offshore wave breaking case (Figure 13i).

683 HEP bathymetry steady currents (right panels of Figure 13) demonstrate a 684 wave-parameter dependence similar to that observed in the LEP bathymetry 685 runs, and SCw intensification above the wreck is again simulated. However, 686 notable differences between the LEP and HEP simulations are also observed.

687 Unlike LEP, in HEP the maxima steady current are located above and 688 downwave of the wreck, with the exception of E5_HEP. In addition, the 689 presence of the recirculation cells increases the magnitude of SCW 690 downwave of the wreck. For the small waves simulation (Figure 13b), a 691 counter clockwise rotation cell forms at the toe of wreck (), a clockwise 692 circulation cell $\left(0.15 \mathrm{~m} \cdot \mathrm{s}^{-1}\right)$ is located in the deepest part of the inshore scour pit, and another counter-clockwise current cell (x 205m) shows a

694 SCw intensity of $0.20 \mathrm{~m} \cdot \mathrm{s}^{-1}$ close to the seabed. In the E2_HEP simulation 
695 (Figure 13d), the SWc velocity is increased to $0.3 \mathrm{~m} \cdot \mathrm{s}^{-1}$ in the centre of the 696 inshore scour pits, and the clockwise rotation current cell at the inshore 697 scour mark $(x \sim 202 \mathrm{~m})$ increases velocity up to $0.3-0.7 \mathrm{~m} \cdot \mathrm{s}^{-1}$. The counter 698 clockwise rotation cell located at $x \sim 207$ reachs up to $0.35 \mathrm{~m} \cdot \mathrm{s}^{-1}$ near the 699 seafloor. In the E3_HEP simulation (Figure 13f) it is notable how the 700 maximum velocity above the wreck extends to the inshore scour pit, and is 701 associated with the larger current cell with clockwise rotation observed 702 above the inshore scour.

703 The breaking process in E4_HEP produces a volume flux in the shoreward 704 direction within water column (see shoreward direction of vector between 4 705 and $5 \mathrm{~m}$ depth in Figure 13h), it restricts the undertow current near to the 706 seabed and intensifies the SCW $\left(0.15 \mathrm{~m} \cdot \mathrm{s}^{-1}-0.20 \mathrm{~m} \cdot \mathrm{s}^{-1}\right)$ around the wreck. 707 In addition, a new current cell develops above the offshore scour pits 708 ( $x \sim 188)$, and weakens the horizontally elongated current cell ( $x \sim 207)$. 

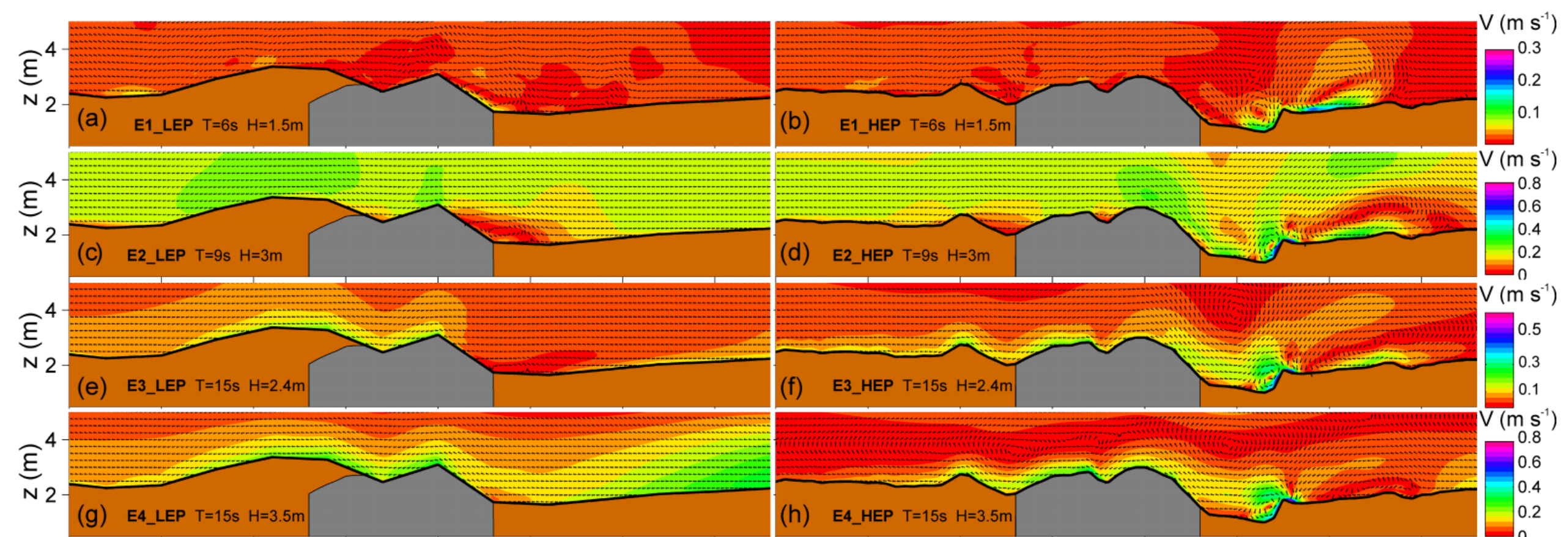
$\mathrm{V}\left(\mathrm{m} \mathrm{s}^{-1}\right)$
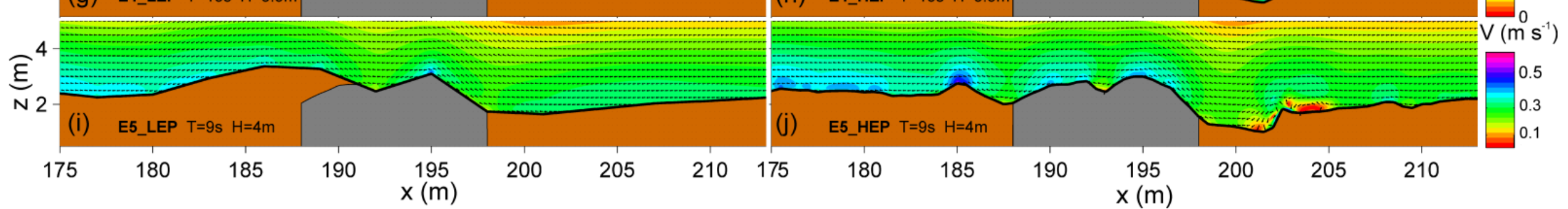

710 Figure 13. Steady current generated by nonlinear effects induced by waves. Left panel shows numerical experiments for LEP conditions.

711 Right panel shows numerical experiment for HEP conditions. Cold colours indicate higher velocities and warm colours indicate lower 712 velocities. 
713 In the E5_HEP simulation, the offshore breaking wave increases SCW

714 current velocity to $0.3 \mathrm{~m} \cdot \mathrm{s}^{-1}$, while the inshore current cell reduces in size 715 and velocity (Figure $13 \mathrm{j}$ ).

\section{$716 \quad 4.6$ Bed shear stress}

717 Live-bed scouring dominates the entire site since the bed shear stress is

718 larger than critical bed shear stress for sediment motion in all wave

719 conditions tested. Figure 14 (a-e) depicts the spatial distribution of the ratio

720 between bed shear stress $(\tau)$ and critical bed shear stress for the sloping

721 bed $\left(\tau_{\mathrm{c}}\right)$, with the bathymetric profiles corresponding to HEP and LEP plotted

722 below (Figure 14f). Note that different scales used for ease of interpretation

723 of the $\tau / \tau_{c}$ features. For all simulations, larger values of $\tau / \tau_{c}$ are obtained at

724 and downwave from the wreck. The maximum $\tau / \tau_{\mathrm{c}}$ occurs downwave of the

725 wreck $(x \sim 200 m)$, it is more evident in the HEP simulations. The $\tau / \tau_{c}$

726 variations induced by bathymetric changes are clear in the larger values of

$727 \tau / \tau_{\mathrm{c}}$ in the LEP simulations at the sand accumulation upwave of the wreck

$728(x=185 m-190 m)$. In addition, the LEP $\tau / \tau_{c}$ maximum occurs around $x=195$

729 at the top of the wreck (Figure 14f), where after a gradual increase the bed

730 shear stress peaks before flow separation. In the HEP simulations

731 bathymetric peaks of $\tau / \tau_{c}$ occur on the offshore scour crest ( $\left.x=185 \mathrm{~m}\right)$, near

732 the centre of the wreck site $(x \sim 192 m)$, just downwave of the top of the

733 wreck ( $x \sim 194.5 \mathrm{~m})$, and at the inshore scour crest ( $x \sim 202.5 \mathrm{~m})$. Notable

734 also are maxima of $\tau / \tau_{c}$ beyond the inshore scour crest $(x=203 m-210 m)$.

735 The increase in wave height and period increases the $\tau / \tau_{\mathrm{c}}$ ratio significantly.

736 Figure $14 d$ shows the $\tau / \tau_{c}$ maximum values for longer and higher waves just

737 before breaking ( $x=205)$, and corresponding to the maximum differences 
738 between LEP and HEP experiments. Finally, for simulations E5_LEP and

739 E5_HEP (Figure $14 \mathrm{e}), \tau / \tau_{\mathrm{c}}$ is reduced due to the drastic reduction of wave

740 height caused by the offshore wave breaking $(x=89 \mathrm{~m})$.
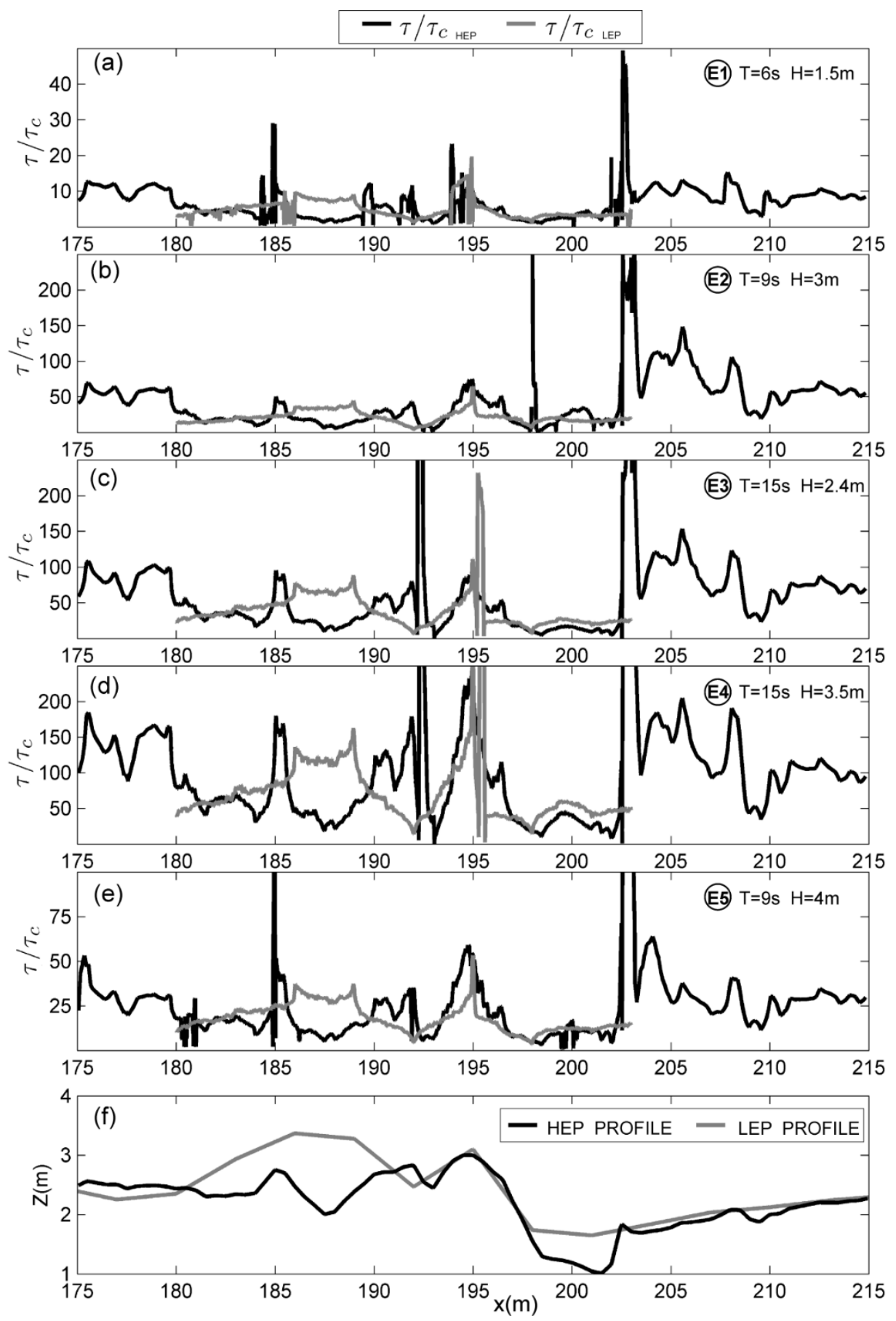

742 Figure 14. Spatial distribution of the ratios between bed shear stress calculated and

743 critical bed shear stress for the sloping bed for the two morphological statuses

744 analyzed. (a) Experiments E1_HEP and E1_LEP. (b) Experiments E2_HEP and 
745 E2_LEP. (c) Experiments E3_HEP and E3_LEP. (d) Experiments E4_HEP and

746 E4_LEP. (e) Experiments E5_HEP E5_LEP. (f) Bathymetric profiles corresponding to 747 HEP and LEP.

\section{Discussion}

749 Wreck-wave interaction and its seasonal variation was analyzed using 750 computational fluid dynamic simulations at the Fougueux site. This method

751 allowed us to evaluate the relative importance of the different processes

752 involved in scouring at a fully-submerged, wave-dominated historic

753 shipwreck site, under the influence of seasonal forcing. Our analysis reveals

754 characteristics of the mean oscillatory flow, flow velocity increase,

755 coherency of structures, recirculation cells and residual turbulence.

756 Although the two bathymetric profiles (LEP and HEP) used in the study were

757 obtained from different sources (multibeam and single beam) and are of

758 different resolution, the variations introduced in the shipwreck shape

759 representation are minor in comparison to the flow pattern variations

760 induced by morphological changes (Figure 1c).

761 The analysis of oscillatory flow for the different wave conditions reveals that

762 seasonal morphological changes largely modify the oscillatory flow velocity

763 pattern around the shipwreck. The inshore and offshore scour observed in

764 HEP simulations promotes the reduction of maximum and minimum near

765 bottom velocity values as a direct consequence of depth increase in both

766 areas (Figures 3 and 4). These modifications occur for all wave conditions,

767 although they are more evident in those experiments with higher $R e$. In

768 addition, the differences in near bed velocity are larger upwave of the hull

769 remains than downwave. This can be attributed to the larger changes in 
770 depth in this location (Figure 1c), and also to the amplification of flow

771 velocity resulting from the erosion of the offshore sand accumulation in

772 HEP, particularly during mean storm conditions (20\%) (Table 2).

\begin{tabular}{|c|c|c|c|c|c|}
\hline Location & $\begin{array}{l}\left\langle U_{m o}\right\rangle_{E 1_{-} H E P} \\
/\left\langle U_{m o}\right\rangle_{E 1_{-} L E P}\end{array}$ & $\begin{array}{l}\left\langle U_{m o}\right\rangle_{E 2 \_H E P} \\
/\left\langle U_{m o}\right\rangle_{E 2 \_L E P}\end{array}$ & $\begin{array}{l}\left\langle U_{m o}\right\rangle_{E 3_{-} H E P} \\
/\left\langle U_{m o}\right\rangle_{E 3_{-} L E P}\end{array}$ & $\begin{array}{l}\left\langle U_{m o}\right\rangle_{E 4_{-} H E P} \\
/\left\langle U_{m o}\right\rangle_{E 4_{-} L E P}\end{array}$ & $\begin{array}{l}\left\langle U_{m o}\right\rangle_{E 5_{-} H E P} \\
/\left\langle U_{m o}\right\rangle_{E 5 \_L E P}\end{array}$ \\
\hline$X=194.5$ & $20 \%$ & $12 \%$ & $14 \%$ & $14 \%$ & $10 \%$ \\
\hline
\end{tabular}

773 Table 2. Flow velocity increase percentage at hull remains $x=194.5$

774 The modification of velocity profiles is promoted by the vortex dynamics. It

775 is observed in the LEP simulations (see for example Figure $3 \mathrm{~b}$ upwave

$776 \mathrm{x}=191$, at $\mathrm{t}^{*}=6 / 9$ and $7 / 9$ and downwave $\mathrm{x}=198$, at $\mathrm{t}^{*}=2 / 9$ and $\left.\mathrm{t}^{*}=4 / 9\right)$.

777 This process is more obvious in HEP simulations, for instance in H2_HEP

778 (see Figures 4b and Figure 8 ). At the toe of hull remains ( $x=198)$ the

779 velocity profile is modified by the counter clockwise vortex ( $t^{*}=4 / 9$ -

$\left.780 t^{*}=7 / 9\right)$, increasing the near bottom velocity. Likewise, the shedding of the

781 vortex shoreward changes the horizontal velocity profiles at $x=205$

$782\left(t^{*}=4 / 9\right)$ and at $x=208.5\left(t^{*}=6 / 9-7 / 9\right)$

783 The dynamics of large coherent structures (LCS) is controlled by the

784 Keulegan-Carpenter (KC) number (Summer, 1991). LCS clearly dominate

785 the HEP simulations due to the presence of inshore and offshore scour-crest

786 systems, which introduce two additional areas of vortex generation. Table 3

787 lists the $\mathrm{KC}$ values for the different locations prone to flow separation and

788 vortex generation: the upwave and downwave scour-crest systems, and the

789 hull remains. For the $\mathrm{KC}$ calculation, the diameter (D) was assumed as the 790 width of the scour-crest, and the distance from the centre of the wreck site 791 to the toe of wreck ( $x \sim 197)$ for the hull remains. 


\begin{tabular}{|c|c|c|c|c|c|c|c|}
\hline & $X(m)$ & $\mathrm{D}(\mathrm{m})$ & E1_LEP & E2_LEP & E3_LEP & E4_LEP & E5_LEP \\
\hline \multirow[t]{2}{*}{ Hull remains } & 195 & 4.1 & 2 & 6 & 12 & 15 & 5 \\
\hline & $X(m)$ & $\mathrm{D}(\mathrm{m})$ & E1_HEP & E2_HEP & E3_HEP & E4_HEP & E5_HEP \\
\hline \multirow{3}{*}{$\begin{array}{l}\text { Hull remains } \\
\text { Offshore } \\
\text { scour-crest } \\
\text { Inshore scour- }\end{array}$} & 195 & 3.7 & 2 & 5 & 12 & 16 & 5 \\
\hline & 185.2 & 2.3 & 3 & 9 & 21 & 26 & 7 \\
\hline & 202.7 & 1.2 & 6 & 7 & 29 & 40 & 16 \\
\hline
\end{tabular}

793 Table 3. KC calculated from numerical experiment of the wave condition

794 tested with LEP and HEP bathymetry.

795 Flow separation does not occur at the wreck or at the offshore scour-crest in

796 simulations E1_LEP and E1_HEP, as was expected for values of $\mathrm{KC}<4$

797 according to Sumer (1991). K and L vortices are only observed at the

798 inshore scour crest $(\mathrm{KC}=6)$, along with the coherent structures $\left(\mathrm{RC}^{+}\right.$and

$799 \mathrm{RC}^{-}$), whose position and rotation match with the recirculation cell identified

800 in Figure 13a.

801 In E2_LEP, flow velocity increases at the wreck $(K C=6)$. As a result type $\mathrm{K}$ 802 and $\mathrm{L}^{\prime}$ vortices are observed for E2_LEP, and the coherent structure $\left(\mathrm{RC}^{+}\right)$

803 is identified as a recirculation cell (Figure 13c). In the E2_HEP simulation, a

804 type $\mathrm{K}^{\prime}$ vortex is formed at the wreck $(\mathrm{KC}=5)$ and remains attached. Vortex 805 shedding does not occur at values of $4<\mathrm{KC}<7$, in agreement with Sumer

806 (1991). At the inshore scour-crest $(K C=7)$, the vortex dynamics are

807 accelerated and the vortex shedding takes place as two vortex pairs; K-L

808 downwave and $M-K_{2}$ upwave. The $M-K_{2}$ pair is segregated, and the $K-L$ pair

809 is transported initially shoreward by the counter clockwise current cell

810 (Figure 13d).

811 Although the $\mathrm{KC}$ increase at the wreck there is no significant variation in the 812 other LEP simulations. 
813 In contrast, in E3_HEP even with the same value of $\mathrm{KC}$ for the wreck, the

814 absence of the offshore sand accumulation promotes the generation of a

815 type $L^{\prime \prime}$ vortex at the centre of the wreck site. The growth of $K C=29$ at the

816 inshore scour-crest does not generate changes in vortex dynamics, solely

817 producing modification in the trajectory of the $M$ vortex that is constrained

818 by the larger clockwise current cell formed above the inshore scour pit

819 (Figure 13f).

820 In the case of the inshore breaking wave E4_HEP, a KC value of 16 is

821 calculated at the wreck, indicating a more complex dynamic as revealed by

822 the presence of a $\mathrm{N}$ vortex developed at the inshore scour pit, the $\mathrm{L}^{\prime}$ vortex

823 split into smaller eddies, and the $L^{\prime \prime}$ vortex at the centre of the wreck site.

824 The vortex $L^{\prime}$ partition could be associated with larger turbulent shear after

825 the wave breaking process (Figure 12d). In addition the clockwise current

826 cell (Figure 13h) marks the trajectory of the M vortex and vortex shedding

827 also takes place at the offshore scour-crest system $(K C=26)$.

828 In simulation E5_HEP, vortices are developed only at the scour-crest

829 system. The two vortex pairs at the inshore scour crest remain attached to

830 the crest, in spite of the fact that the value $K C=16$ is larger than the

831 supposed threshold for vortex shedding (KC=7) (Sumer, 1991).

833 It is important to note how vortex dynamics modifies the velocity profiles

834 and increases the turbulent shear. This is observed when mean streamwise

835 velocity (Figure 3 and Figure 4) and turbulent shear profiles (Figure 11 and

836 12) in different wave phases are compared with the position of vortices at

837 those phases (Figures 6-10). This promotes an increase in boundary layer 
838 thickness (Figure 5), similar to the one that forms in the presence of sand

839 ripples when they originate from rhythmic vortices (Nielsen, 1992).

840 On the subject of turbulent shear stress, results show an important

841 increment in turbulence for the HEP morphological setting. This behaviour is

842 observed in all simulations, especially downwave of the wreck, with flow

843 velocity increase, LCS, and recirculation cells developed downwave.

844 The recirculation cells generated by the SCW are only present in the LEP

845 simulations at the toe of the wreck, with velocities lower than $0.10 \mathrm{~m} . \mathrm{s}^{-1}$.

846 Higher velocities of steady current are associated with undertow, which is

847 markedly significant in the cases of breaking wave E4_LEP and E5_LEP

848 (Figure 13). The recirculation cells are observed in all HEP simulations,

849 particularly downwave and with larger velocities $\left(0.3-0.7 \mathrm{~m} . \mathrm{s}^{-1}\right)$ than in LEP.

850 The mean oscillatory flow brings sediment into suspension and as the

851 results have shown controls the trajectory of LCS increasing its effect in the

852 sediment transport.

853 It is remarkable the contribution of the LCS to the SCW. It can be observed 854 in the cases of E2_HEP, E3_HEP and E4_HEP (Figures 8, 9 and 10), where

855 the major LCS located in the inshore scour contributes to the residual

856 velocity as point the recirculation cell located in that area. (Figures $13 d, 13 f$

857 and $13 \mathrm{~h}$ ). In addition, when the LCS are weak i.e. E1_HEP (Figure 6), the

858 recirculation cells can be identified by the Okubo-Weiss criteria. In that

859 case, at difference of the vortex, the RC remains during the entire wave

860 period with slight variations in its position (Figure 13a).

861 These processes modify the spatial distribution of bed shear stress on the 862 wreck and the surrounding seabed. Thus the speed up and vortex shedding 
863 and its associated turbulence produce a maximum in $\tau / \tau_{c}$ downwave of the 864 wreck, more evident in HEP simulations (Figure 14, x 200). The increase in 865 bed shear stress caused by the vortex and the shear stress underneath the 866 vortex is clearly observable for the HEP simulation shoreward of the inshore 867 scour $(x>202)$, and at the toe of the wreck (Figure $14 \mathrm{~b})$. For more energetic 868 conditions (Figure $14 c$ and Figure $14 d$ ) higher values of $\tau / \tau_{c}$ are found in 869 LEP simulations, revealing that the influence of frictional velocity 870 amplification due to depth reduction in LEP is larger than the influence 871 caused by the effect of turbulence underneath the vortex in the HEP 872 simulations. This effect is more conspicuous upwave of the wreck, at the 873 offshore sand accumulation in LEP. Furthermore, the increase in the grid 874 size of the first computational cell above the seabed outside of region two in 875 the computational mesh (R2) (Figure 2) induces an error in the bed shear 876 stress calculation. This is observed in HEP experiments (Figure 14), where $877 \tau / \tau_{\mathrm{c}}$ changes in the limit of the R2 region. However, the results are valid for 878 HEP because the relative changes induced by the increase in the grid size of 879 the first computational cell above the seabed outside of region two (R2), are 880 smaller than the changes caused by the hydrodynamic and vortex pattern.

881 On the contrary, in the case of LEP the changes promoted by the 882 hydrodynamics and the vortex pattern are not meaningful in comparison 883 with those induced by the variation in mesh size. Therefore, in the case of 884 LEP bathymetry the relative magnitude of this error with respect to the 885 spatial variation of $\tau / \tau_{c}$ invalidates the $\tau / \tau_{c}$ results outside the region $R 2$.

886 Previous investigations at the wreck site (Fernández-Montblanc et al., 887 2016), based on time-lapse bathymetric surveys and conventional 888 hydrodynamic modelling, concluded that sediment is alternatively deposited 
889 and eroded at the Fougueux site in response to seasonal wave climate

890 variation. Thus the morphological changes observed in HEP and LEP are

891 intrinsically linked to the seasonal wave climate variation in an inter-annual

892 interactive cycle. Furthermore, a feedback mechanism occurs where the 893 geomorphological changes induce changes in the oscillatory flow velocity 894 patterns around the shipwreck. This iterative cycle can be described as 895 follows. During the mean storm after a prolonged low energy period (from 896 May to September) (H1_LEP) the friction velocity and speed of flow 897 produces the erosion of the offshore sand accumulation and the initial 898 development of the onshore scour mark. The development of the inshore 899 and offshore scour marks increases the speed of flow on the hull remains 900 (see Table 2), accelerates the vortex dynamics and the intensification of the 901 current cell, resulting in amplification of the shear stress. It occurs most 902 notably during the extreme events (H2_HEP, H3_HEP, H4_HEP and 903 H5_HEP), leading to the enhancement of sediment transport by shoreward 904 vortex shedding. Finally, during the swell-wave period (low-energy 905 conditions from May to September) asymmetry between the orbital 906 velocities beneath crests and troughs tend to drive sediment from the 907 offshore direction towards the coast producing the offshore sand 908 accumulation, partially filling partially the inshore scour mark with the 909 surrounding sediment. 


\section{Conclusions}

916 The CFD simulations from the Fougueux site indicate that the friction

917 velocity due to oscillatory flow and flow velocity amplification are the

918 dominant physical processes in operation during the low energy period, and

919 the large coherent structure formed at the toe of the wreck initiates scour at

920 that location. During the high energy period, the relevance of turbulent

921 shear and the large coherent structure are markedly greater, and flow

922 increases at the wreck site. In addition, the recirculation cells originated by

923 steady streaming and undertow are more important in high energy periods.

924 The velocity increase of the current cell in high energy settings modifies the

925 trajectory of the shed vortex increasing its capacity of sediment transport.

926 The results confirm CFD as a valuable tool to understand the mechanisms

927 promoting scouring at shipwreck sites and other submerged structures

928 under the influence of waves. Beyond the aim of this study, the successful

929 application of a full scale CFD study in the simulation of waves and structure

930 interactions allows the behaviour of these structures in full scale and

931 realistic morphological conditions to be tested. In addition, the full scale

932 CFD application will allow the parameterization of different physical process

933 related to waves, thus avoiding the many restrictions of physical lab

934 experiments and field-based deployments during extreme conditions.

935 In summary, the full scale CFD modelling presented in this study allows

936 detailed analysis of key physical processes in coastal engineering,

937 addressing limitations inherent in traditional physical models and field

938 deployments. 


\section{Acknowledgements}

940 This work has been supported by the Spanish Ministry of Economy and

941 Competitiveness, Project CTM2010-16363 (ARQUEOMONITOR).

\section{References}

943 Astley, A., Dix, J.K., Thompson, C., Sturt, F., 2014. A seventeen year, near-

944 annual, bathymetric time-series of a marine structure (SS Richard

945 Montgomery). In: Cheng, L., Draper, S. and An, H. (Eds.), Scour and

946 Erosion: Proceedings of the 7th International Conference on Scour and

947 Erosion. International Conference on Scour and Erosion. Taylor \& Francis, 948 pp. $715-724$.

949 Baeye, M., Quinn, R., Deleu, S., Fettweis, M., 2016. Detection of shipwrecks 950 in ocean colour satellite imagery. J. Archaeol. Sci. 66, 1-6.

951 Baldock, T.E., 2006. Long wave generation by the shoaling and breaking of

952 transient wave groups on a beach. Proceedings of Royal Society A, 462, 953 1853-1876.

954 Bates, C.R., Lawrence, M., Dean, M., Robertson, P., 2011. Geophysical 955 Methods for Wreck-Site Monitoring: the Rapid Archaeological Site Surveying 956 and Evaluation (RASSE) programme. Int. J. Naut. Archaeol. 40, 404-416.

957 Bergstrand, T., Godfrey,N.I., 2007. Reburial and analyses of archaeological 958 remains: Studies on the effect of reburial on archaeological materials 959 performed in Marstrand, Sweden 2002-2005. The RAAR project.

960 Kulturhistoriska dokumentationer nr 20. Bohusläns Museum: Udevalla. 
961 Bozorgnia, M., Eftekharian, A., Jiin Jen, L., 2014. CFD modelling of a

962 solitary wave overtopping breakwater of varying submergence. Coastal

963 Engineering Proceedings, 1(34), waves 7.

964 Chang, K.A., Liu, P.L.F., 1999. Experimental investigation of turbulence 965 generated by breaking waves in water of intermediate depth. Phys. Fluids.

$96611,3390-3400$.

967 De Hauteclocque, G., Dix., J, Lambkin, D., Turnock, S., 2007. Flow and 968 Likely Scour Around Three Dimensional Seabed Structures Evaluated Using 969 RANS CFD, University of Southampton Ship science report, 144, 90 pp.

970 Dixen, M., Sumer, B. M., Fredsøe, J., 2013. Numerical and experimental

971 investigation of flow and scour around a half-buried sphere. Coast. Eng. 73, 972 84-105.

973 Elhmaïdi, D., Provenzale, A., Babiano, A., 1993. Elementary topology of 974 two-dimensional turbulence from a Lagrangian view point and single particle 975 dispersion. J. Fluid Mech. 257, 533-558.

976 Fernández-Montblanc, T., Quinn, R., Izquierdo, A., Bethencourt, M., 2016.

977 Evolution of a shallow water wave-dominated shipwreck site: Fougueux 978 (1805), Gulf of Cadiz. Geoarchaeology. 31, 487-505.

979 Gracia, F.J., Rodríguez Vidal, J., Cáceres, L.M., Belluomini, G., Benavente, 980 J., Alonso, C. (2008). Diapiric uplift of an MIS 3 marine deposit in SW 981 Spain: Implications for Late Pleistocene sea level reconstruction and 982 palaeogeography of the Strait of Gibraltar. Quat. Sci. Rev. 27, 2219-2231.

983 Higuera, P., Lara, J.L. and Losada, I.J., 2013. Realistic wave generation and 984 active wave absorption for Navier-Stokes models: Application to OpenFOAM. 985 Coast. Eng. 71,102-118. 
986 Hussain, A. K. M. F., 1983. Coherent structures Reality and myth. Phys.

987 Fluids. 26, 2816-2850.

988 Isern-Fontanet, J., Font, J., García-Ladona, E., E, M., Millot, C., Taupier-

989 Letage, I., 2004. Spatial structure of anticyclonic eddies in the Algerian

990 basin (Mediterranean Sea) analyzed using the Okubo-Weiss parameter.

991 Deep Sea Res. Part II Top. Stud. Oceanogr. 51(25-26), 3009-3028.

992 Jensen, B. L., Sumer, B. M., Fredsøe, J., 1989. Turbulent oscillatory

993 boundary layers at high Reynolds numbers. J. Fluid Mech. 206, 265-297.

994 Jeong, J., Hussain,F. (1995). On the identification of a vortex. J. Fluid Mech. $995285,69-94$.

996 Leino, M., Ruuskanen, A. T., Flinkman, J., Kaasinen, J., Klemela, U. E.,

997 Hietala, R., Nappu, N., 2011. The Natural Environment of the Shipwreck

998 Vrouw Maria (1771) in the Northern Baltic Sea: an assessment of her state

999 of preservation. Int. J. Naut. Archaeol. 40, 133-150.

1000 Le Méhauté, B., 1976. Introduction to Hydrodynamics and Water Waves.

1001 Springer-Verlag, New York.

1002 Longo, S., M. Petti, Losada, I.J., 2002. Turbulence in the swash and surf 1003 zones: a review. Coast. Eng. 45(34), 129-147.

1004 Longuet-Higgins, M.S., Stewart, R., 1960. Change in the form of short 1005 gravity waves on long waves and tidal currents. J. Fluid Mech. 8, 565-583.

1006 Mattioli, M., A. Mancinelli, Brocchini, M., 2013. Experimental investigation of 1007 the wave-induced flow around a surface-touching cylinder. J. Fluids Struct. $1008 \quad 37,62-87$. 
1009 McNinch, J.E., Wells, J.I., Trembanis, A.C., 2006. Predicting the fate of 1010 artefacts in energetic, shallow marine environments: an approach to site 1011 management. Int. J. Naut. Archaeol. 35, 290-309.

1012 Melville, W.K., Veron, F., White, C.J., 2002. The velocity field under 1013 breaking waves:coherent structures and turbulence. J. Fluid Mech. 454, $1014203-233$.

1015 Menter, F. R., 1994. Two-Equation Eddy-Viscosity Turbulence Models for 1016 Engineering Applications. AIAA Stud. J., (32)8, 1598-1605.

1017 Murray, S.P., 1970. Settling velocities and vertical diffusion of particles in 1018 turubulent water 1970. J. Geophys. Res. (75) 9, 1647-1654.

1019 Nadaoka, K., Hino, M., Koyano,k Y., 1989. Structure of the turbulent flow 1020 field under breaking waves in the surf zone. J. Fluid Mech. 204, 359-387.

1021 Nelson, J.M., Shreve, R.L., McLean, S.R., Drake, T.G., 1995. Role of near1022 bed turbulence structure in bed load transport and bed form mechanics. 1023 Water Resour. Res. 31, 2071-2086.

1024 Nielsen, A., Sumer, B. M., Ebbe, S., Fredsøe, J., 2012. Experimental Study 1025 on the Scour around a Monopile in Breaking Waves. J. Waterw. Port C1026 ASCE. 138(6), 501-506.

1027 Nielsen, P., 1992. Coastal Bottom Boundary Layers and Sediment 1028 Transport. World Scientific, 324 pp.

1029 Pasquero, C., Provenzale, A., Babiano, A., 2001. Parameterization of 1030 dispersion in two-dimensional turbulence. J. Fluid Mech. 439, 279-303.

1031 Quinn, R., 2006. The role of scour in shipwreck site formation processes and 1032 the preservation of wreck-associated scour signatures in the sedimentary 
1033 record - evidence from seabed and sub-surface data. J. Archaeol. Sci. 33, 1034 1419-1432.

1035 Quinn, R., Boland, D., 2010. The role of time-lapse bathymetric surveys in 1036 assessing morphological change at shipwreck sites. J. Archaeol. Sci. 37, 1037 2938-2946.

1038 Quinn, R., Smyth, T.A.G., in press, Processes and patterns of flow, erosion, 1039 and deposition at shipwreck sites: a computational fluid dynamic simulation. 1040 Archaeol. Anthropol. Sci., https://doi.org/10.1007/s12520-017-0468-7.

1041 Rodríguez Mariscal, N.E., Rieth, E., Izaguirre, M., 2010. Investigaciones en 1042 el pecio de Camposoto: hacia la identificación del navío francés Fougueux, 1043 Revista PH del Instituto Andaluz del Patrimonio Histórico, 75, 94-107.

1044 Ruuskanen, A. T., Kraufvelin, P., Alvic, R., Díaz, E.R., Honkonen, J., 1045 Kanerva, J., Karell, K., Kekäläinen, P., Lappalainen, J., Mikkola, R., 1046 Mustasaari, T., Nappu, N., Nieminen, A., Roininen, J., Svahnbäck, K., 2015. 1047 Benthic conditions around a historic shipwreck: Vrouw Maria (1771) in the 1048 northern Baltic proper. Cont. Shelf Res. 98, 1-12.

1049 Saunders, R.D., 2005.Seabed scour emanating from submerged three 1050 dimensional objects: Archaeological case studies'. PhD Thesis, Department 1051 of Civil and Environmental Engineering, University of Southampton, UK. 1052 214p.

1053 Sawamoto, M., Yamashita,T., Kitmura, T., 1982. Measurements of 1054 turbulence over vortex-ripple. Coastal Engineering Proceedings; No 18 1055 (1982): Proceedings of 18th Conference on Coastal Engineering, Cape 1056 Town, South Africa, 1982. 
1057 Scott, C.P., Cox, D.T., Maddux, T.B., Long, J.W., 2005. Large-scale

1058 laboratory observations of turbulence on a fixed barred beach. Meas. Sci.

1059 Technol. 16, 1903-1912.

1060 Smyth, T.A.G., Quinn, R., 2014. The role of computational fluid dynamics in

1061 understanding shipwreck site formation processes. J. Archaeol. Sci. 45,

$1062 \quad 220-225$.

1063 Soulsby, R., 1997. Dynamics of Marine Sands: A manual Practical

1064 Applications. Thomas Telford Telford, London.

1065 Stansby, P. K., Feng, T., 2005. Kinematics and depth-integrated terms in

1066 surf zone waves from laboratory measurement. J. Fluid Mech. 529, 279-

1067310.

1068 Sumer, B. M., Fredsøe, J., 1990. Scour below pipelines in waves. J. Waterw.

1069 Port C-ASCE. 116(3), 307-323.

1070 Sumer, B. M., Jensen, B. L., Fredsøe, J., 1991. Effect of a plane boundary

1071 on oscillatory flow around a circular cylinder, J. Fluid Mech. 225, 271-300.

1072 Sumer, B., Christiansen, N., Fredsøe, J., 1993. Influence of Cross Section

1073 on Wave Scour around Piles. J. Waterw. Port C-ASCE. 5(477), 477-495.

1074 Sumer, B.M., Christiansen, N., Fredsøe, J., 1997. The horseshoe vortex and

1075 vortex shedding around a vertical wall-mounted cylinder exposed to waves.

1076 J. Fluid Mech. 332, 41-70

1077 Sumer, B.M., Whitehouse, R.J.S., Tørum, A., 2001. Scour around coastal

1078 structures: a summary of recent research. Coast. Eng. 44, 153-190. 
1079 Sumer, B.M., Fredsøe, J., 2002. The Mechanics of Scour in the Marine 1080 Environment. World Scientific Publishing Co Pte Ltd. Advanced Series on 1081 Ocean Engineering, no. 17.

1082 Sumer, B.M., Fredsoe, J.R., Lamberti, A., Zanuttigh, B., Dixen, M., Gislason, 1083 K., Di Penta, A.F., 2005. Local scour at roundhead and along the trunk of 1084 low crested structures. Coast. Eng. 52, 995-1025.

1085 Testik, F.Y., Voropayev, S.I., Fernando, H.J.S., 2005. Flow around a short 1086 horizontal bottom cylinder under steady and oscillatory flows. Phys. Fluids. 1087 17, 47-103.

1088 Ting, F. C.; Kirby, J. T., 1996. Dynamics of surf-zone turbulence in a spilling 1089 breaker. Coast. Eng. 27(3-4), 131-160.

1090 Torres-Freyermuth, A., Lara, J., Losada, I., 2010. Numerical modelling of 1091 short and long-wave transformation on a barred beach. Coast. Eng. 57,3171092330.

1093 Voropayev, S.I., Testik, F.Y., Fernando, H.J.S., Boyer, D.L., 2003. Burial 1094 and scour around short cylinder under progressive shoaling waves. Ocean 1095 Eng. 30, 1647-1667.

1096 Ward, I. A. K., Larcombe, P., Veth, P., 1999. A New Process-based Model 1097 for Wreck Site Formation. J. Archaeol. Sci. 26, 561-570.

1098 Whitehouse, R.J.S., Sutherland, J., Harris, J., 2011. Evaluating scour at 1099 marine gravity foundations. Proceeding ICE e Maritime Engineering, 164 $1100 \quad(4), 143-157$.

1101 Wheeler, A. J., 2002. Environmental Controls on Shipwreck Preservation: 1102 The Irish Context. J. Archaeol. Sci. 29(10), 1149-1159. 
1103 Young, D.M., Testik, F.Y., 2009. Onshore scour characteristics around

1104 submerged vertical and semicircular breakwaters. Coast. Eng. 56, 868-875.

1105 